\title{
SPECTRAL IRRADIANCE CALIBRATION IN THE INFRARED. XIII. "SUPERTEMPLATES" AND ON-ORBIT CALIBRATORS FOR THE SIRTF INFRARED ARRAY CAMERA
}

\author{
MarTin COHEN \\ Radio Astronomy Laboratory, 601 Campbell Hall, University of California at Berkeley, Berkeley, CA 94720; \\ mcohen@astro.berkeley.edu \\ S. T. Megeath \\ Harvard-Smithsonian Center for Astrophysics, 60 Garden Street, Cambridge, MA 02138; tmegeath@cfa.harvard.edu \\ Peter L. Hammersley and Fabiola Martín-Luis \\ Instituto de Astrofísica de Canarias, Calle Vía Láctea, E-38200 La Laguna, Tenerife, Spain; plh@1l.iac.es, \\ fabi@1l.iac.es \\ AND \\ JOHN STAUFFER \\ SIRTF Science Center, 314-6, California Institute of Technology, 1200 East California Boulevard, \\ Pasadena, CA 91125; stauffer@ipac.caltech.edu \\ Received 2002 November 19; accepted 2003 January 24
}

\begin{abstract}
We describe the technique that will be used to develop a set of on-orbit calibrators for the Infrared Array Camera (IRAC) and demonstrate the validity of the method for stars with spectral types either K0-M0 III or A0-A5 V. For application to SIRTF, the approach is intended to operate with all available optical, nearinfrared (NIR), and mid-infrared (MIR) photometry and to yield complete absolute spectra from UV to MIR. One set of stars is picked from Landolt's extensive network of optical (UBVRI) calibrators, the other from the Carter-Meadows set of faint IR standards. Traceability to the "Cohen-Walker-Witteborn" framework of absolute photometry and stellar spectra is assured. The method is based on the use of either "supertemplates," which represent the intrinsic shapes of the spectra of K0-M0 III stars from far-UV $(1150 \AA)$ ) to MIR $(35 \mu \mathrm{m})$ wavelengths, or Kurucz synthetic spectra for A0-A5 V stars. Each supertemplate or Kurucz model is reddened according to the individual star's extinction and is normalized using available characterized optical photometry. This paper tests our capability to predict NIR (JHK) magnitudes using supertemplates or models constrained by Hipparcos/Tycho or precision ground-based optical data. We provide absolutely calibrated $0.275-35.00 \mu \mathrm{m}$ spectra of 33 Landolt and Carter-Meadows optical standard stars to demonstrate the viability of this technique, and to offer a set of IR calibrators 100-1000 times fainter than those we have previously published. As an indication of what we can expect for actual IRAC calibration stars, we have calculated the absolute uncertainties associated with predicting the IRAC magnitudes for the faintest cool giant and hot dwarf in this new set of calibration stars.
\end{abstract}

Key words: infrared radiation — methods: analytical — stars: late-type — techniques: spectroscopic

On-line material: color figure, response curves, template files

\section{INTRODUCTION}

This series of papers on spectral irradiance calibration in the infrared was motivated by the need to establish accurate celestial flux standards for use in astronomical spectroscopy and photometry by spaceborne, airborne, and groundbased instruments. The earlier papers in this series present the foundations of a method for establishing an entire allsky network of IR flux calibrators. The near- to mid-IR imager on NASA's Space Infrared Telescope Facility (SIRTF) - the Infrared Array Camera (IRAC) - has four detector arrays, with central wavelengths of 3.6, 4.5, 5.8, and $8.0 \mu \mathrm{m}$. The IRAC detectors are roughly 2000 times more sensitive than those of IRAS and would saturate on all but the faintest stars of the existing network. In the current paper, we develop the methodology to predict the IR inband fluxes of fainter stars using optical photometry, and we report the results of an experiment to demonstrate the reliability of this method. Consequently, this paper describes the approach we are pursuing to create the onorbit cool giant and hot dwarf calibrators for IRAC and will serve as a proof of concept by validating the efficacy of the method.

The objectives of the current paper were to select a mix of cool giants and A dwarfs with well-characterized, precision optical photometry; determine accurate optical spectral types for all these stars; represent their energy distributions by appropriately reddened stellar photospheric spectra; normalize these energy distributions using the optical bands; predict $J H K$ from the normalized spectra; secure characterized $J H K$ observations of as many of these stars as possible; compare observed and predicted near-IR (NIR) magnitudes; and create a set of absolute spectra for stars considerably fainter than those of our previous all-sky network. The final products to be generated by this effort will likewise be complete, continuous, absolutely calibrated spectra from the UV to mid-IR (MIR), consistent with the context described in Paper X (Cohen et al. 1999).

Section 2 overviews the basic components of our methodology, treating the construction of combined optical-IR "supertemplates" of cool, normal giant stars, which extend from 0.115 to $35 \mu \mathrm{m}$; the optical-IR atmospheric models 
and their synthetic spectra for the hot A dwarfs; and the reddening corrections applied to the selected stars. Section 3 follows with a demonstration of the method using a set of selected Landolt and Carter-Meadows standards with published, precision $U B V R I$ magnitudes, for which we secured our own optical classification spectroscopy, and applies the technique to the prediction of NIR and MIR magnitudes from optical photometry and spectra. Section 4 describes the results of renormalizing these supertemplate and model spectra using all available optical, NIR, and MIR photometry and describes how to obtain these spectra. Section 5 sets our work in context with the Two Micron All Sky Survey (2MASS) and SIRTF, showing how this method will be used to estimate the in-band fluxes of our potential calibrators in the four IRAC bands and assessing the degree to which this approach will satisfy the absolute calibration goals for IRAC. In an appendix, we detail the extension of the method to accommodate space-based optical data from Hipparcos/Tycho, and we focus on the problems encountered with Tycho-1 photometry of cool stars, which are largely mitigated by Tycho- 2 data.

\section{METHODOLOGY}

\subsection{Overview}

The method extends previously created, empirical, absolutely calibrated, 1.2-35 $\mu \mathrm{m}$ composite spectra ("templates") of bright stars with spectral types of K0 III to M0 III into the UV and optical. These optical-to-IR "supertemplates" are used as generic spectral shapes for all stars of a given spectral type and luminosity class. The supertemplates, after they have been reddened using a standard extinction law and normalized to broadband optical and NIR/MIR photometric measurements, serve as proxy spectra for a network of stars that are too faint or too numerous to be effectively measured individually.

\subsection{Limitations of the Current Network of Stars}

The current network of 422 stars was constructed by fitting 1.2-35 $\mu \mathrm{m}$ templates to photometry from ground-based and spaceborne telescopes (Paper X). The fundamental reference standards for the spectra are absolutely calibrated models of the A-type stars Sirius and Vega. The templates were constructed by combining empirical spectral fragments for eight bright $\mathrm{K} / \mathrm{M}$ giants obtained from ground-based, airborne, and spaceborne telescopes. The absolute calibration of these spectra was determined by taking the ratios of the cool stars' spectra to the observed spectrum of either Vega (below $13 \mu \mathrm{m}$ ) or Sirius and then scaling the ratioed spectra by the theoretical A star spectra, degraded to the actual spectral resolution of the observations.

The range of fluxes in the network of 422 templates decreases from 800 to $5 \mathrm{Jy}$ in the IRAS $12 \mu \mathrm{m}$ band, with a handful of stars as faint as $1.1 \mathrm{Jy}$ in a new, unpublished set of 602 templates by Walker \& Cohen (2002), which includes the 422 stars of Paper X, sometimes with updated versions of their spectra. The Midcourse Space Experiment (MSX) satellite has recently provided a validation of the relative and absolute calibration of subsets of the stars that compose this network. Cohen et al. (2001) have analyzed independently calibrated $M S X$ photometry in six bands from 4.3 to $21.3 \mu \mathrm{m}$ from all three tiers of the network - Vega/Sirius, the bright $\mathrm{K} / \mathrm{M}$ giants, and the fainter template stars - and find that the $M S X$ data agree with the predicted fluxes to within the estimated uncertainties.

To provide meaningful calibrators for IRAC's four bands requires flux densities from about 1 Jy down to about $1 \mathrm{mJy}$ in these bands. However, we wish to maintain traceability to our absolute spectral products that support the Infrared Telescope in Space (Murakami et al. 1996), the Diffuse Infrared Background Experiment (Hauser et al. 1998, their Table 1; Cohen 1998), MSX (Mill et al. 1994; Egan et al. 1999; Price et al. 2001), and ESA's Infrared Space Observatory (ISO; Kessler et al. 1996) instruments. To extend $\mathrm{K} 0$ M0 III's from $12 \mu \mathrm{m}$ flux densities of $5 \mathrm{Jy}$ down to the requisite values for IRAC necessitates using stars that are fainter by about 9 mag than those of the current network, at the level of $V \sim 11-12$. One way to achieve even fainter IR flux densities, for stars with the same visual magnitudes as the current network, is to use hot stars, for which Kurucz model atmospheres are quite reliable in the optical and IR.

\subsection{Creating New" Templates" and "Supertemplates" for $K / M$ Giants}

The original set of spectral types for which Cohen et al. (1995, 1996a, 1996b) constructed complete, empirical 1.2$35 \mu \mathrm{m}$ spectra consists of $\mathrm{K} 0, \mathrm{~K} 1.5, \mathrm{~K} 3, \mathrm{~K} 5$, M0, M1.5, M2.5, and M3.4 III. To minimize any potential variability, we have concentrated solely on the range $\mathrm{K} 0-\mathrm{M} 0$ III for IRAC calibrators. To create a finer grid of templates, we have interpolated these spectra to create new IR templates for types $\mathrm{K} 1, \mathrm{~K} 2, \mathrm{~K} 4$, and $\mathrm{K} 7 \mathrm{III}$.

The interpolation of a template file, with its five elements ( $\lambda, F_{\lambda}$, absolute error in $F_{\lambda}$, and local and global biases; see Paper $\mathrm{X}$ ) first requires removal of the global and local biases from the total uncertainty, leaving the solely random component of the uncertainty. Two templates whose spectral types flank that of the required type are normalized in $\lambda^{4} F_{\lambda^{-}}$ space so that they lie on top of one another longward of the $\mathrm{SiO}$ fundamental. The flux density, random error, local bias, and global bias are linearly interpolated using the $s$-parameter of de Jager \& Nieuwenhuijzen (1987), which represents spectral type more continuously and linearly than either stellar effective temperature or its logarithm for normal, mature stars. Finally, the local and global biases are recombined in quadrature with the random errors, yielding the quantities for a new template file. This procedure provides a more complete set of template spectra to apply to new candidate calibrators, and a set that is fully consistent with our published, complete, empirical spectra of bright $\mathrm{K} / \mathrm{M}$ giants. Figure 1 illustrates the newly created K4 III template derived by interpolation between K3 III ( $\alpha$ Hya) and K 5 III ( $\alpha$ Tau) templates.

To establish the credibility of such interpolated products, we compare the K4 III template with consistently calibrated spectral observations of $\beta$ UMi (K4 III) taken on the Kuiper Airborne Observatory (KAO) using the HIFOGS instrument (Witteborn et al. 1995) and by the IRAS Low Resolution Spectrometer (LRS). Both HIFOGS (Cohen et al. 1995) and the LRS (Cohen, Walker, \& Witteborn 1992b) have been calibrated in an identical context to that of Paper $\mathrm{X}$. The star $\beta$ UMi was observed on the KAO flights of 1995 April 14 and 19 and calibrated using identically taken HIFOGS spectra of $\alpha$ Boo, matching the air mass of $\beta \mathrm{UMi}$ as closely as possible. Figure 2 illustrates this direct comparison. Note that the two spectrometers have very different 


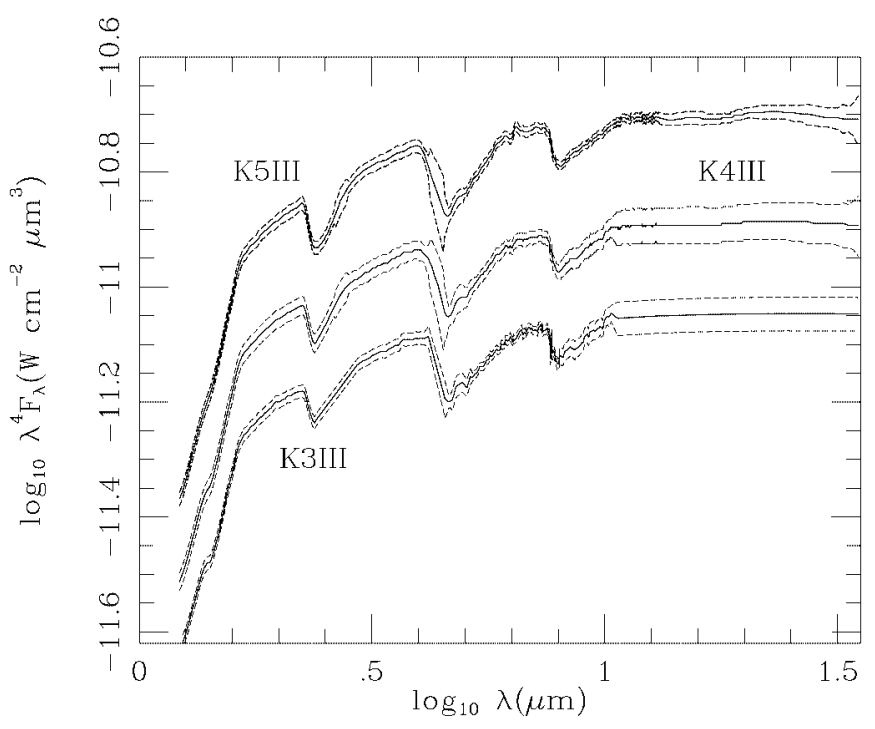

FIg. 1.-Infrared template of a K4 III derived by interpolation between the observed spectra of $\alpha$ Hya (K3 III) and $\alpha$ Tau (K5 III). Solid lines represent the spectra; dashed lines show the $\pm 1 \sigma$ bounds.

resolving powers, of order 200 for HIFOGS $(4.9-9.6 \mu \mathrm{m})$ and $20-50$ for the LRS $(7.7-22.7 \mu \mathrm{m})$. This accounts for the divergence, for 7.7-8.5 $\mu \mathrm{m}$, between the filled (KAO) and open (LRS) squares in the deep $\mathrm{SiO}$ fundamental absorption. With these exceptions, there is satisfactory overlap at the 1-2 $\sigma$ level between observations and the K4 III template, corresponding to agreement within $5 \%$ (except in the saturated ozone feature at $\sim 9.3 \mu \mathrm{m}$ in the KAO spectrum).

To construct combined optical-to-IR "supertemplates," it is necessary to extend the standard $1.2-35 \mu \mathrm{m}$ calibrated spectra into the optical. This step was accomplished by splicing the IR templates to the average of the several spectra for stars with relevant MK spectral types from Pickles's (1998) spectral library that extends from the far-UV (1150 $\AA)$ to the NIR $(2.5 \mu \mathrm{m})$. In some cases, it was necessary to fill

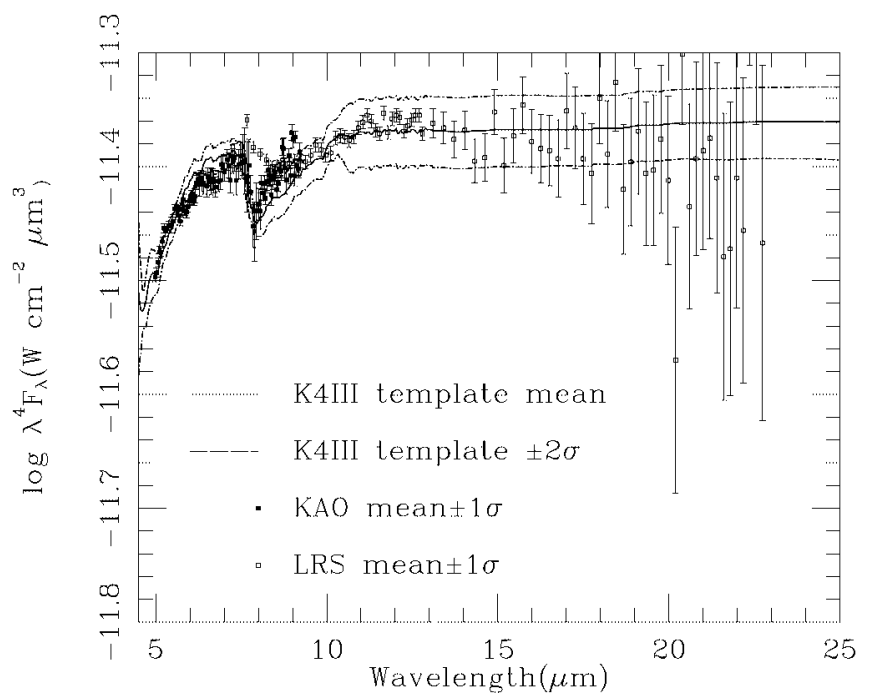

FIG. 2.-Comparison of the K4 III template with KAO ( filled squares; resolving power $\sim 200$ ) and LRS (open squares; resolving power 25-50) spectral observations of the $\mathrm{K} 4$ giant $\beta$ UMi. The solid line is $\mathrm{K} 4$ III template; dot-dashed lines show the $\pm 2 \sigma$ bounds on the template. The observed spectral points carry $\pm 1 \sigma$ error bars.

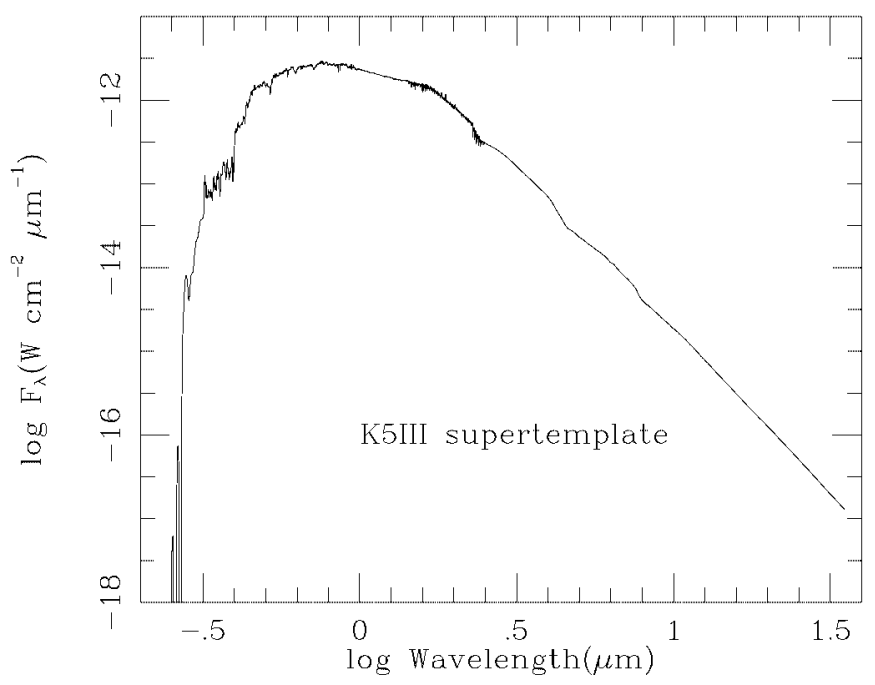

FIG. 3.-Complete K5 III supertemplate, combining Pickles's optical K5 III spectrum with our observed infrared spectrum of $\alpha$ Tau.

in "gaps" in the Pickles "UVKLIB" spectra of the cool giants and to rectify erroneous blackbody-based interpolations by using our own fully observed spectra longward of $1.22 \mu \mathrm{m}$. When the final products were deemed complete, accurate, and without discontinuities, the observed $B-V$ colors for these supertemplates were synthesized and compared with the literature to check that they satisfactorily represented reddening-free spectral shapes, within the respective uncertainties. Figure 3 presents the K5 III supertemplate from 0.27 to $35 \mu \mathrm{m}$. Supertemplates formally extend down to $0.115 \mu \mathrm{m}$, but cool giants rarely are well detected by IUE below $0.27 \mu \mathrm{m}$, so we offer them only longward of $0.275 \mu \mathrm{m}$, for practical purposes. Each Pickles spectrum was itself constructed as the average of spectra from up to 17 other libraries, suggesting probable robustness as a representative for each type.

Although we created a K7 III template, we recognize that these are rather rarely encountered, and further, Pickles (1998) does not include this type in his spectral library. Therefore, we simply averaged his spectra for K5 III and M0 III and appended that to our interpolated K7 III IR template.

Implicit in these constructs is the assumption that whatever the actual metallicity and, more importantly, the abundances of $\mathrm{C}, \mathrm{O}$, and $\mathrm{Si}$ are for any individual star, we can apply a generic spectral shape whose heritage is partly traceable to the bright stars observed spectroscopically from $\mathrm{KAO}$, from the ground, and from space (using the LRS). The reason for this assumption is that this same technique will be applied to visually very faint stars for which it is most unlikely that such detailed information will be available.

\subsection{Atmospheric Models and Synthetic Spectra of the Hot Stars}

The A dwarf synthetic spectra were all taken from the standard, solar abundance grid of Kurucz LTE model atmospheres (Kurucz 1993a, 1993b) and correspond to stars of effective temperature 9795, 9397, 9016, 8710,8433, and $8185 \mathrm{~K}$, for the set A0 V to A5 V, respectively (de Jager \& Nieuwenhuijzen 1987), and with gravity, $\log g$, between 4.16 and 4.25 (varying monotonically with type). 


\subsection{Supertemplates: Validations and Usage}

Analysis of both empirical IR spectra and theoretical models indicates that effective temperature exerts the dominant influence on the shapes of these spectra, with gravity and especially metallicity much less significant. Tests carried out on Kurucz models (for hot, warm, and even cool stars) during the calibration work in support of ISO showed that differences arose between IR templates and models, even when the literature provided explicit estimates of temperature, gravity, and $[\mathrm{Fe} / \mathrm{H}]$. However, empirical and model spectra were generally in good agreement, within $\pm 2 \%$ in the IR continuum, and less than $5 \%$ in molecular bands (e.g., the $\mathrm{CO}$ first-overtone bands). Further, those differences that do arise were not dependent on $[\mathrm{Fe} / \mathrm{H}]$ but rather on a star's individual abundances of C, O, and $\mathrm{Si}$. Such data are not generally available for faint stars, even when an estimate of $[\mathrm{Fe} / \mathrm{H}]$ might exist.

Another way of validating this entire process derives from the direct comparison of observed stellar angular diameters and "radiometric" diameters (Paper X). There is excellent agreement between measured and radiometrically predicted stellar angular diameters, with the implication that continua and even molecular band shapes and depths are well matched at least to first order. This result also lends validity to the supertemplates.

\subsection{Reddening Corrections}

Stellar reddening was determined by comparing Landolt's (1992) measured $B-V$ (or, equivalently, the same indexes measured by Carter \& Meadows 1995) with the mean intrinsic colors described below. If a star proved too blue for its type, we assigned zero to $A_{V}$, otherwise we assigned $3.10 E(B-V)$. The resulting extinctions were applied to the intrinsic supertemplates.

\subsubsection{Intrinsic $B-V$ Colors}

The natural spread of observed $B-V$ indexes for unreddened stars of any given spectral type is quite substantial, attaining about \pm 0.15 (J.-C. Mermilliod 1993, private communication). We attribute this to intrinsic cosmic scatter among the stars, caused by variations in metallicity; the individual abundances of elements - primarily $\mathrm{C}, \mathrm{O}$, and $\mathrm{Si}$ - whose lines and bands significantly sculpt the stellar energy distribution and are not governed solely by temperature and gravity; the quantization of a continuum of stellar temperatures and gravities into discrete spectral classes; and errors in the assignment of spectral types drawn from the literature. It is for this latter reason that we have mounted our own optical spectroscopy program (see $\S 3.4$ ).

To exact the greatest precision from our technique requires the best estimates for the mean color indexes of K0-M0 III and A0-A5 V stars, because optically faint (and hence IR-faint) stars of both these types are likely to be significantly reddened. For these estimates, we chose to rely on the Hipparcos output database (van Leeuwen 1997). Individual records offer the Hipparcos team's best value of $B-V$ color, along with kinematic and parallax data, spectral types, and a host of information so that one can readily reject multiple and variable stars and objects with poorly determined parallaxes. The catalog enables the determination of observed colors from subsets with as many as 50-200 stars for each populous type of $\mathrm{K}$ giant, and at least 50 A dwarfs per subclass, even when demanding meaningful measurements of parallax (and hence distance) at the 5-10 $\sigma$ level.

We converted observed $B$ and $V$ magnitudes to absolute $M_{B}$ and $M_{V}$ and into estimates of each star's intrinsic $B-V$. If a star's distance, calculated from the reciprocal of a significant parallax, placed it within the dust-free Local Bubble (Fitzgerald 1970; Perry \& Johnston 1982; Perry, Johnston, \& Crawford 1982), then we assigned zero extinction on the basis of the extent of the dust-free zone. For stars beyond $75 \mathrm{pc}$, we assigned an $A_{V}$ of $0.625 \mathrm{mag} \mathrm{kpc}^{-1}$ (and $A_{B} /$ $\left.A_{V}=1.299\right)$ to determine the absolute magnitudes and intrinsic $B-V$. The values used for the extinction per kiloparsec, and for the ratio $A_{B} / A_{V}$, come from the reddening law described below. Because we test each parallax-based distance against a simplistic representation of the Local Bubble, and then average the resulting extinctions (many of which are zero), any residual effects of Lutz-Kelker bias are greatly diluted.

\subsubsection{The Reddening Law}

The representation of the actual law of extinction that we used between far-UV and MIR was based on the seventhorder polynomial fits suggested by Cardelli, Clayton, \& Mathis (1989) for the UV and far-UV, and the eighth-order polynomial fits of O'Donnell (1994) for the optical-to-NIR range, and it joins smoothly onto the law of reddening used in Cohen (1993) longward of $4.7 \mu \mathrm{m}$. We note that there are few substantive differences at short wavelengths $(\leq 1.0 \mu \mathrm{m})$ between different, empirically derived, reddening laws in the literature. This overall law compares favorably, for example, with the representations of other authors, such as Fluks et al. (1994), whose IR reddening law is actually derived from theoretical considerations. We have likewise compared our reddening curve with that described by Fitzpatrick (1999), finding excellent accord except in the vicinity of the $R$ band (see Fitzpatrick's discussion of this region) and, of course, throughout the interstellar silicate absorptions that are absent from his representation of the IR extinction curve. However, Fitzpatrick's (1999) quantification of the uncertainties in UV-optical extinction curves is particularly valuable. To estimate uncertainties in the NIR and MIR, we first compared the law described above with the independently derived law of Rieke \& Lebofsky (1985). Following their adoption of uncertainties of $\sim 15 \%$ in $A_{\lambda} / A_{V}$, we find that the differences between our values and those of the 21 Rieke-Lebofsky points lie well within $1 \sigma$ (combined) for 18 wavelengths. Only one point (at $13 \mu \mathrm{m}$ ) lies more than $2.5 \sigma$ away. These quantitative uncertainties in our reddening curve are now accommodated in the code that generated the actual spectra of the Landolt and CarterMeadows stars in this paper and will likewise be used to create spectra of the SIRTF calibrators.

\section{DEMONSTRATION AND PROOF OF CONCEPT}

\subsection{Overview}

We now offer a demonstration of our methodology using a set of selected optical standard stars with published, precision UBVRI magnitudes, and for which we have undertaken an optical spectroscopy program at Mount Hopkins Observatory to establish their spectral types. Mount Hopkins spectral types and extinctions based on the observed $B-V$ are used to appropriately redden the 
supertemplates or Kurucz synthetic spectra to represent each selected star's energy distribution. These shapes are then normalized by $U B V R I$ photometry and the resulting spectra used to predict IR $J H K$ magnitudes. Direct comparison between well-characterized NIR photometry and these predictions tests the efficacy of our approach.

\subsection{Selection of Stars}

We now describe our chosen stars, which were drawn from two different sets, with their own independent optical and NIR photometry, and discuss the sources of their spectral types. We offer cross-checks on two independent $U B V R I$ data sets, compare space-based and ground-based optical normalizations of supertemplates, and demonstrate consistent predictions of different systems of $J H K$ for one star common to the two data sets.

Our primary optical data set is the precision UBVRI photometry on stars in the Kapteyn Selected Areas offered by Landolt $(1973,1983,1992)$. These furnish an abundant set of faint optical standards measured through well-documented passbands. Only about 600 of the many stars observed by Landolt had ever been classified spectroscopically, by Drilling \& Landolt (1979). Of these, only about 30 were suggested to be K0-M0 giants. Early experiments with the technique described in this paper indicated that some of these types were surely suspect by several subclasses. Consequently, we selected 32 alleged cool giants and about an equal number of stars lying within $10^{\circ}$ of the ecliptic plane, whose $B-V$ colors suggested that they might be K0-M0 III's, for a program of optical classification spectroscopy. In total, we investigated 62 Landolt stars.
Another valuable set of test objects is the collection of stars offered by Carter \& Meadows (1995) as faint standards for 3-4 m class ground-based telescopes. Carter \& Meadows also provide their own $U B V R I$ measurements from the South African Astronomical Observatory (SAAO) $0.5 \mathrm{~m}$ telescope at Sutherland. To test the A stars, we decided to apply our method to all stars for which Carter \& Meadows cite a spectral type between "A0" and "A5," even when a luminosity class is lacking. We had hoped to demonstrate the plausibility of assigning dwarf luminosities to these stars on the basis of a successful prediction of their NIR magnitudes. This gave us an additional 19 stars to investigate.

We have augmented the Landolt sample of cool giants for which we obtained Mount Hopkins spectra with SA 94-251, SA 108-475, and HD 197806 (whose type of K0 III, cited by Carter \& Meadows, is vindicated by Houk \& Swift 1999).

We found a final subset of 24 cool giants stars to be useful: that is, their actual spectral types fell into our desired spectral ranges, we were able to secure precision NIR photometry from Tenerife or from Carter \& Meadows, and we could accurately predict their $J H K$ magnitudes. These 24 stars appear in Table 1. The other Landolt stars were "lost" either because we found them to be $\mathrm{K}$ or $\mathrm{M}$ dwarfs or because we were unable to secure Tenerife $J H K$ data for them.

We extracted the most accurate coordinates that could be found either in SIMBAD ("S") or in the Guide Star Cata$\log$, version 1.3, with corrections from ACT ("G"), for these stars. Searching SIMBAD by any of a host of possible star names is not equivalent to searching by coordinates, as D. Shupe, who kindly extracted these coordinates for our first observing run, noted for SA 114-670. The SIMBAD

TABLE 1

ICRS 2000.0 Coordinates for Those Landolt SA Stars Found to Be Useful for Our Purposes

\begin{tabular}{|c|c|c|c|c|c|c|}
\hline $\begin{array}{l}\text { Star } \\
\text { Name }\end{array}$ & R.A. & Decl. & Source & $B$ & $V$ & $\begin{array}{l}\text { Spectral } \\
\text { Type }\end{array}$ \\
\hline SA 92-336 ................. & 005501.401 & +004722.39 & $\mathrm{~S}$ & 9.03 & 8.05 & K0 III \\
\hline SA 94-251 .................. & 025746.98 & +00 1602.7 & G & 12.45 & 11.22 & K1 III \\
\hline 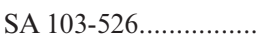 & 115654.19 & -003013.8 & $\mathrm{G}$ & 11.99 & 10.90 & K0 III \\
\hline 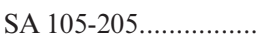 & 133552.598 & -005753.56 & $\mathrm{~S}$ & 10.16 & 8.80 & K3 III \\
\hline SA $105-405 \ldots \ldots \ldots \ldots$ & 133559.535 & -003439.62 & $\mathrm{~S}$ & 9.83 & 8.31 & K5 III \\
\hline SA 107-35 .................... & 153728.856 & -005305.44 & S & 9.05 & 7.78 & K2 III \\
\hline 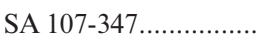 & 153835.773 & -003557.74 & $\mathrm{~S}$ & 10.74 & 9.44 & K1.5 III \\
\hline 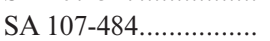 & 154016.80 & -002114.5 & G & 12.55 & 11.31 & K3 III \\
\hline SA $108-475 \ldots \ldots \ldots \ldots \ldots$ & 163700.09 & -003440.0 & $\mathrm{~S}$ & 12.69 & 11.31 & K3 III \\
\hline 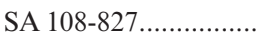 & 163721.171 & -002448.59 & $\mathrm{~S}$ & 9.27 & 7.96 & K2 III \\
\hline SA 108-1918 ................. & 163750.12 & -000036.1 & G & 12.82 & 11.38 & K2/K3 III \\
\hline SA 109-231.................. & 174519.965 & -002551.60 & $\mathrm{~S}$ & 10.79 & 9.33 & K2 III \\
\hline 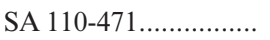 & 184126.680 & +003351.88 & $\mathrm{~S}$ & 8.92 & 7.47 & K2/K3 III \\
\hline SA $112-275 \ldots \ldots \ldots \ldots \ldots$ & 204235.426 & +000720.23 & $\mathrm{~S}$ & 11.12 & 9.91 & K0 III \\
\hline 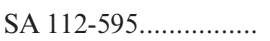 & 204118.47 & +001628.3 & G & 12.95 & 11.35 & M0 III \\
\hline 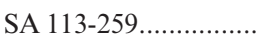 & 214144.83 & +001740.2 & $\mathrm{G}$ & 12.94 & 11.74 & K2 III \\
\hline 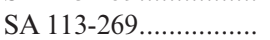 & 214201.322 & +001745.22 & $\mathrm{~S}$ & 10.59 & 9.48 & K0 III \\
\hline 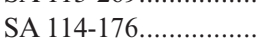 & 224310.183 & +002115.53 & $\mathrm{~S}$ & 10.72 & 9.24 & K4/K5 III \\
\hline 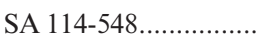 & 224136.85 & +005906.1 & G & 12.96 & 11.60 & K2.5 III \\
\hline 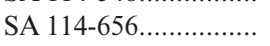 & 224135.2 & +011110 & $\mathrm{~S}$ & 13.61 & 12.64 & K1 III \\
\hline 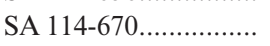 & 224209.310 & +011016.78 & M & 12.31 & 11.10 & $\mathrm{~K} 1 / \mathrm{K} 2 \mathrm{III}$ \\
\hline 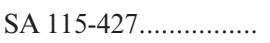 & 234314.430 & +010647.01 & $\mathrm{~S}$ & 10.03 & 8.86 & K2 III \\
\hline SA $115-516 \ldots \ldots \ldots \ldots \ldots$ & 234415.374 & +011412.65 & $\mathrm{~S}$ & 11.46 & 10.43 & K1.5 III \\
\hline HD 197806.................... & 204730.773 & -431033.63 & $\mathrm{~S}$ & 10.84 & 9.66 & K0 III \\
\hline
\end{tabular}

NotE.-Source codes: (S) SIMBAD when queried by star name; (G) Guide Star Catalog 1.3; (M) SIMBAD when queried by coordinates. Spectral types are our own. Units of right ascension are hours, minutes, and seconds, and units of declination are degrees, arcminutes, and arcseconds. 
position returned by this name is considerably in error compared with the true coordinates that correctly return the star under the name PPM 700910. Table 1 summarizes accurate coordinates, with Landolt or Carter-Meadows $B$ and $V$ magnitudes. Carter \& Meadows provide rough J2000 positions of their stars, but their A dwarfs are all HD stars and precise coordinates are available unambiguously in the literature.

\subsection{Optical Photometric Bands}

Professor A. Landolt kindly provided digitized versions of the tables from his 1992 paper in which are given details of the CTIO UBVRI passbands used for his photometry of optical standard stars, along with the response curves of the photomultipliers. We further multiplied the product of each passband and the detector response curve by a mean atmospheric transmission spectrum appropriate to Cerro Tololo Inter-American Observatory (CTIO), in Chile. To assess the intervening telluric transmissions above CTIO, we represented the atmospheric transmission using PLEXUS, ${ }^{1}$ an Air Force Research Laboratory-validated "expert system" that incorporates atmospheric code, specifically, MODTRAN 3.7, SAMM, and FASCODE3P with the HITRAN98 archives. PLEXUS contains an extensive database to support its expert aspect, so that the effects of Rayleigh scattering, aerosols, and particulates appropriate to the desert conditions of the CTIO sites were included. Paper X also used PLEXUS calculations to represent the site-specific atmospheric transmissions necessary to represent the tens of ground-based filters characterized in that work, so we have maintained consistency in our treatment of all systems, both optical and IR. We did compare the CTIO atmospheric transmission from PLEXUS with the simpler formulation for extinction given by Hayes \& Latham (1975), based explicitly on the effects of Rayleigh scattering, absorption by ozone, and scattering by aerosols, and we found good accord.

We created $U B V R I$ relative spectral response curves (RSRs) from the combinations of filter, atmosphere, and detector profiles that were normalized to peak values of unity. There have been several comparisons of the SAAO UBVRI system with Johnson's UBVRI (Cousins 1984; Bessell 1979) and CTIO VRI (Menzies 1989), and a widerranging discussion is given by Straižys (1992). Menzies (1989) found slight differences between the Cousins RI passbands and those of Landolt, while Menzies et al. (1991) concluded that the SAAO and Landolt CTIO $U B V$ bands differ from one another and probably also from Johnson's. We have attempted to pursue the actual bands in use at SAAO by way of the detailed response functions offered by Straižys

${ }^{1}$ See http://www2.bc.edu/ sullivab/soft/plexus.html.
(1992, who also cites Bessell 1979, 1983), but this has proved unsatisfactory. We further note the following statement by Menzies (1989): " The photometer on the 0.5-m telescope in Sutherland is in continuous use so there has not been an opportunity to determine the filter transmission functions experimentally."

Consequently, we have represented the Carter-Meadows relative response curves (filter plus atmosphere plus detector) in $U B V R I$ by the same response curves as those published by Landolt (1992). Direct comparison of UBVRI for objects in common to the Carter-Meadows and Landolt samples justifies this step, as exemplified by SA 94-251 and SA 108-475 (Table 2). For the former, the differences between magnitudes measured in both systems are within the assigned $3 \sigma$ uncertainties; for the latter, all differences are within the $1 \sigma$ uncertainties.

Menzies et al. (1991) based their conclusions on a set of 212 Landolt stars for which they present SAAO UBVRI measurements. These stars span a wide range of spectral types. We have carried out the same experiment, but restricted to 21 Landolt stars in common, for which our Mount Hopkins spectra indicate K0-M0 III spectral types. The result of comparing Landolt-minus-SAAO magnitudes, using inverse-variance weighting for the combinations, shows, for UBVRI, respectively, $-0.002 \pm 0.022$, $-0.012 \pm 0.011, \quad-0.005 \pm 0.007, \quad-0.011 \pm 0.009$, and $-0.011 \pm 0.010$. On the basis of this restricted range of spectral types relevant to our needs, we conclude that there are no significant differences between Landolt and SAAO photometry. This conclusion also enables us to utilize the data in Table 1 of Menzies et al. (1991) with our subset of Landolt stars. For simplicity, when we later refer to "Landolt" or "BVRI photometry," this might include both Landolt's and Menzies' measurements.

All five system response curves were then integrated over our standard, calibrated, Kurucz model spectrum of Vega (Cohen et al. 1992a) to provide their "zero-magnitude attributes." These attributes (Table 3 ) include the in-band flux (irradiance) and its uncertainty and the monochromatic specific intensity (i.e., $F_{\lambda}$ ), as well as the isophotal wavelength for Vega of each filter. For convenience we have also incorporated $F_{\nu}$, the monochromatic specific intensity in frequency terms, in units of janskys. Note that the isophotal $F_{\nu}$ cannot be accurately rendered simply as $\left(\lambda_{\text {iso }}[\mu \mathrm{m}]\right)^{2}$ $\times\left(F_{\lambda}\left[\mathrm{W} \mathrm{cm} \mathrm{cm}^{-2} \mu \mathrm{m}^{-1}\right]\right) /\left(3 \times 10^{-16}\right)$, because of the finite bandwidths of these filters. We have recast each RSR in frequency terms and have performed the same integrals for in-band, bandwidth, and $F_{\nu}$ as we carried out for $F_{\lambda}$. The on-line version of Table 3 offers these combined RSRs via the links in the "Filter" column.

While we (e.g., Paper X) have always defined zero magnitude in the infrared by this Kurucz spectrum of Vega, this star does not have zero magnitudes in the optical. We

TABLE 2

Comparison of Landolt and Carter-Meadows $U B V R I$ Data for Two Stars in Common

\begin{tabular}{|c|c|c|c|c|c|c|}
\hline Star & System & $U$ & $B$ & $V$ & $R$ & $I$ \\
\hline \multirow[t]{2}{*}{ SA 94-251 ................ } & Landolt & $13.704 \pm 0.0036$ & $12.423 \pm 0.0015$ & $11.204 \pm 0.0010$ & $10.545 \pm 0.0013$ & $9.957 \pm 0.0015$ \\
\hline & Carter-Meadows & $13.750 \pm 0.054$ & $12.454 \pm 0.016$ & $11.224 \pm 0.006$ & $10.572 \pm 0.009$ & $9.986 \pm 0.009$ \\
\hline \multirow[t]{2}{*}{ SA $108-475 \ldots \ldots \ldots \ldots . . .}$. & Landolt & $14.151 \pm 0.005$ & $12.689 \pm 0.002$ & $11.309 \pm 0.0014$ & $10.565 \pm 0.001$ & $9.900 \pm 0.002$ \\
\hline & Carter-Meadows & $14.165 \pm 0.020$ & $12.703 \pm 0.020$ & $11.314 \pm 0.013$ & $10.580 \pm 0.018$ & $9.916 \pm 0.016$ \\
\hline
\end{tabular}


TABLE 3

Absolute Calibration of the Ground-based Optical Photometry Systems Supporting This Work

\begin{tabular}{|c|c|c|c|c|c|c|c|}
\hline Filter & $\begin{array}{l}\text { In-Band Flux } \\
\left(\mathrm{W} \mathrm{cm}^{-2}\right)\end{array}$ & $\begin{array}{l}\text { In-Band Uncertainty } \\
\qquad\left(\mathrm{W} \mathrm{cm}^{-2}\right)\end{array}$ & $\begin{array}{l}\text { Bandwidth } \\
(\mu \mathrm{m})\end{array}$ & $\begin{array}{c}F_{\lambda} \text { (iso) } \\
\left(\mathrm{W} \mathrm{cm}^{-2} \mu \mathrm{m}^{-1}\right)\end{array}$ & $\begin{array}{c}\lambda(\text { iso }) \\
(\mu \mathrm{m})\end{array}$ & $\begin{array}{c}F_{\nu} \\
(\mathrm{Jy})\end{array}$ & $\begin{array}{c}\mathrm{AB}_{\nu} \\
(\mathrm{mag})\end{array}$ \\
\hline Landolt $U \ldots \ldots \ldots \ldots \ldots$ & $2.823 \mathrm{E}-13$ & $4.353 \mathrm{E}-15$ & 0.0711 & $3.971 \mathrm{E}-12$ & 0.3745 & 1649 & +0.857 \\
\hline Landolt $B \ldots \ldots \ldots \ldots \ldots$ & $5.371 \mathrm{E}-13$ & $8.242 \mathrm{E}-15$ & 0.0819 & $6.562 \mathrm{E}-12$ & 0.4481 & 4060 & -0.121 \\
\hline Landolt $V \ldots \ldots \ldots \ldots \ldots$ & $3.327 \mathrm{E}-13$ & $5.086 \mathrm{E}-15$ & 0.0878 & $3.789 \mathrm{E}-12$ & 0.5423 & 3723 & -0.027 \\
\hline Landolt $R \ldots \ldots \ldots \ldots$ & $3.860 \mathrm{E}-13$ & $5.772 \mathrm{E}-15$ & 0.1697 & $2.274 \mathrm{E}-12$ & 0.6441 & 3168 & +0.148 \\
\hline Landolt $I \ldots \ldots \ldots \ldots \ldots . . . .$. & $1.438 \mathrm{E}-13$ & $2.159 \mathrm{E}-15$ & 0.1274 & $1.129 \mathrm{E}-12$ & 0.8071 & 2459 & +0.423 \\
\hline
\end{tabular}

NoтE.-Quantities tabulated correspond to the definition of zero magnitude in each filter.

adopted magnitudes for Vega of $U=0.024, B=0.028$, $V=0.030, R=0.038$, and $I=0.034$ (Bessell, Castelli, \& Plez 1998). Therefore, we derived the true zero-magnitude irradiance and isophotal $F_{\lambda}$-values from those of our Vega spectrum, brightening the Vega values accordingly. Table 3 lists the resulting zero-magnitude attributes for the five bands consistent with our previously published absolute calibrations in the optical and IR. Landolt's system of photometry is based on Johnson's magnitudes and consequently corresponds to the above magnitudes for Vega. Therefore, we have applied no zero-point offsets to align his photometry with our definition of the zero-magnitude attributes.

The final column of Table 3 gives the monochromatic $\mathrm{AB}_{\nu}$ magnitudes, as defined by Oke \& Gunn (1983), that correspond to zero magnitude for each of the five bands (sometimes described as "zero-point magnitudes").

\subsection{Our Classification Spectroscopic Program}

Our 62 selected Landolt stars were observed with the FAST spectrograph on the $1.5 \mathrm{~m}$ Tillinghast Telescope of the Fred Lawrence Whipple Observatory (Fabricant et al. 1998). The spectrograph was configured to provide 4000 $7000 \AA$ spectra with 5 A resolution. The stars were observed as part of a queue observing program on eight different nights, under conditions ranging from photometric to thin clouds, at air masses below 1.64. Exposure times ranged from 0.5 to $1800 \mathrm{~s}$. A $2^{\prime \prime}$ slit was used to obtain $5 \mathrm{~A}$ resolution. The data were dark-subtracted and flat-fielded, and one-dimensional spectra were extracted. These spectra were then subjected to background subtraction using the adjacent off-source spectra in the original two-dimensional spectra, and a spike rejection routine was applied to eliminate cosmic-ray hits. The wavelength scale was determined using calibration lamps.

To establish a methodology for spectral types that was consistent with the MK classification system, spectra of 34 MK standards (24 cool giants, eight cool dwarfs, and two cool supergiants, drawn from the Perkins revised types of Keenan \& McNeil 1989) were obtained in parallel with the targeted 62 Landolt objects, using an identical instrument setup and data reduction procedure. These spectra, displayed in Figures 4 and 5, show that the FAST spectra, when ordered by spectral type and luminosity class, exhibit a systematic progression of spectral features. This demonstrates that accurate spectral classification consistent with the MK system can be obtained with FAST spectra. Based on independent types in the two wavelength regions, 4000 5500 and 5500-7000 $\AA$, at our $\sim 5 \AA$ resolution we estimate the uncertainty in our assigned types to be better than \pm 1 spectral subclass.

The release of Volume 5 of the Michigan Catalogue of Two-dimensional Spectral Types for the HD Stars (Houk \& Swift 1999) extends the previous four southern hemisphere volumes across the celestial equator. This systematic reclassification in the MK system has enabled us to compare our Mount Hopkins types for 14 of the brighter Landolt stars
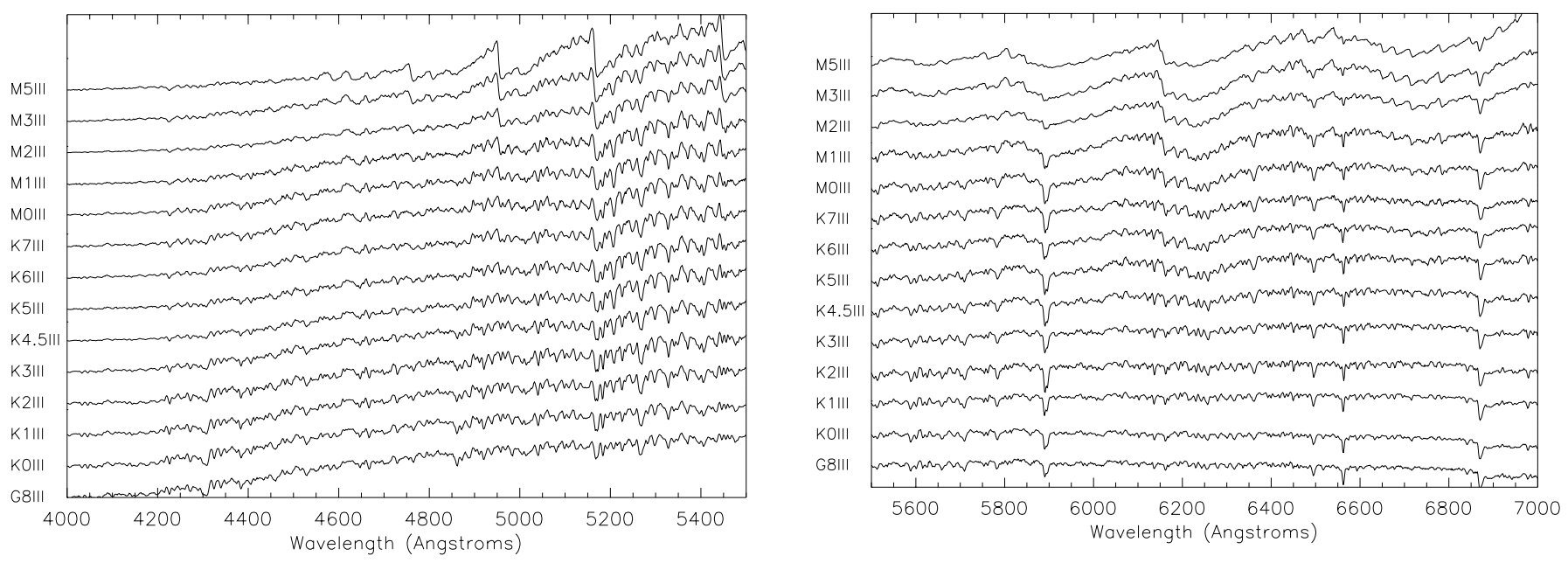

FIG. 4.-FAST spectra of giant stars with spectral types taken from the catalog of Keenan \& McNeil (1989). Although the entire 4000-7000 A range was observed in a single exposure, the spectra are displayed in two sections: 4000-5500 $\AA$ (left) and 5500-7000 $\mathrm{A}$ (right). These plots show a clear and pronounced progression of numerous spectral features with spectral type. 

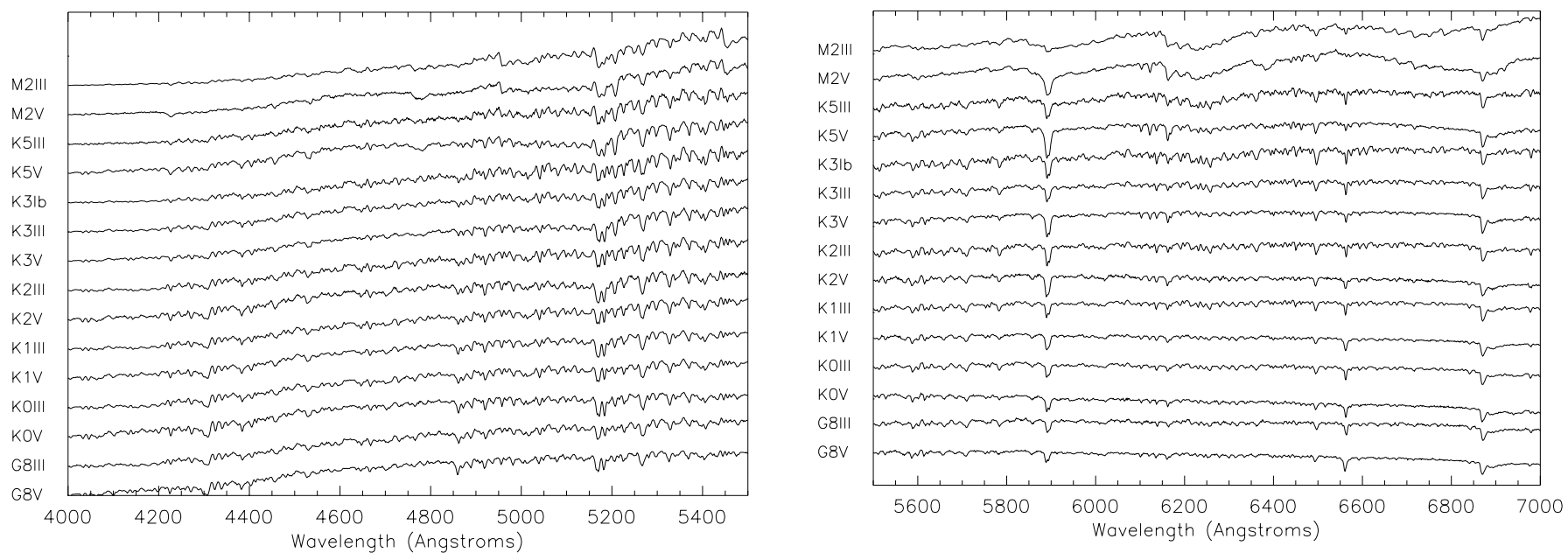

FIG. 5.-FAST spectra of dwarf, giant, and supergiant stars with spectral types ranging from G8 to M2. The luminosity classes and spectral types were taken from the catalog of Keenan \& McNeil (1989). As in Fig. 4, the spectra are displayed in two plots spanning 4000-5500 A (left) and 5500-7000 $\AA$ $($ right). Both ranges show features with an obvious luminosity dependence; in particular, the Ca I line at $6122 \AA$ and the Fe II feature at $5169 \AA$.

that have HD numbers with the Michigan types. For these $\mathrm{K}$ and $\mathrm{M}$ giants, our types do not differ from the Michigan types by more than 1 subclass, validating our estimated uncertainty in spectral type. Furthermore, in classifying the Landolt cool giants we have become particularly aware of the relevance of the Michigan philosophy whereby one assigns, for example, a type of "K2/K3 III" as opposed to K2.5 III, with the implication that the star in question does not resemble in every way an MK type of K2.5 III but, rather, that different criteria are found in the spectrum, some characteristic of a K2 III and others of a K3 III.

Volume 5 of the Michigan Catalogue now offers twodimensional types for many of the Carter-Meadows A stars, a number of which are indeed assigned to dwarf luminosity class, the bulk of them indicating agreement (to within 1 subclass) with the types Carter \& Meadows (1995) drew from the literature.

\subsection{Predicting NIR Flux Densities \\ 3.5.1. New NIR Photometry of Faint Standards}

To test the efficacy of predicting NIR magnitudes from these optical data, we secured JHK photometry using the $1.5 \mathrm{~m}$ Telescopio Carlos Sánchez (TCS), ${ }^{2}$ at Izana, Tenerife. Paper X (in its Table 2 under "Tenerife," and Table 3 under "P. H.") presents the zero-point offsets and absolute attributes of this well-characterized set of filters. Table 4 presents our new NIR photometry for the Landolt stars that we have so far been able to measure from Tenerife, together with their uncertainties. All stars but one were measured on at least two nights in at least one observing period (either 1998 May 23-24 or 1999 July 12-14), and we obtained good accord between data from the two separate observing runs (Table 4). Data for stars observed at two epochs have been combined using inverse-variance weighting, and the combined data also appear in Table 4. The alternative route depends on

\footnotetext{
2 The TCS is operated on the island of Tenerife by the Instituto de Astrofísica de Canarias at the Spanish Observatorio del Teide of the Instituto de Astrofísica de Canarias.
}

Carter-Meadows $J H K$ data from SAAO, an equally well characterized photometric system, also detailed by Paper $\mathrm{X}$ (in its Table 2 under "SAAO," and Table 3 under "B. C.").

For each hot stellar model or cool stellar supertemplate deemed relevant to a particular star, we assembled all available precision optical photometry, whether due to Landolt, Carter-Meadows, or Menzies. We then integrated all five $U B V R I$ response curves over the reddened spectrum (or the two [Tycho only] or three [Tycho and Hipparcos] spacebased optical response curves likewise; see Appendix). The resulting integrals through each system band provide the inband flux, and converting the observed input magnitude into an equivalent irradiance through Table 3 , we derive a scale factor for each filter in order to match the observed and normalized irradiance values. Each overall set of optical data (five or 10 ground-based and/or two or three spacebased magnitudes) yields an inverse-variance-weighted mean scale factor for the reddened supertemplate (using the photometric uncertainties that constitute an essential element of our approach) and a fractional uncertainty in this mean multiplier, termed the "supertemplate bias" and expressed as a percentage of the mean scale factor. We limit the magnitude uncertainties to be $\geq 0.005$, for all optical and NIR observations, to avoid any single filter's overwhelming the weighted scale factor. Once the supertemplate bias is available, this quantity is combined in quadrature with the global bias in the original supertemplate shape and thus finds its way into the total wavelength-dependent errors for any star.

Table 5 illustrates the process of determining the scale factor for a reddened supertemplate for the star SA 112-275, a K0 III with $A_{V}=0.620$. The first column indicates the filter used, the second column gives the scale factor to match predicted to observed in-band fluxes, the third column shows the fractional uncertainty in that scale factor, and the last column provides the isophotal wavelength associated with the combination of filter and supertemplate. The final row summarizes the inverse-variance-weighted mean scale for this star based on the five individual scale factors. Note that this table is based solely on Landolt $U B V R I$, whereas 
TABLE 4

New TCS $J H K$ Photometry and UnCERTAinties For LANDOLt Stars

\begin{tabular}{|c|c|c|c|c|c|c|c|c|}
\hline Star & Date & $J$ & $H$ & $K$ & $\epsilon_{J}$ & $\epsilon_{H}$ & $\epsilon_{K}$ & $\begin{array}{c}\text { No. Chop } \\
\text { Cycles }\end{array}$ \\
\hline SA $92-336 \ldots \ldots \ldots \ldots \ldots$ & 1999 Jul 12-14 & 6.325 & 5.828 & 5.741 & 0.014 & 0.016 & 0.016 & 4 \\
\hline SA $103-526 \ldots \ldots \ldots \ldots \ldots$ & 1998 May 23-24 & 8.993 & 8.467 & 8.372 & 0.015 & 0.008 & 0.011 & 7 \\
\hline SA $105-205 \ldots \ldots \ldots \ldots \ldots$ & 1998 May 23-24 & 6.320 & 5.628 & 5.491 & 0.013 & 0.008 & 0.006 & 6 \\
\hline SA $105-405 \ldots \ldots \ldots \ldots \ldots$ & 1998 May 23-24 & 5.532 & 4.793 & 4.626 & 0.017 & 0.011 & 0.011 & 6 \\
\hline \multirow[t]{3}{*}{ SA $107-35 \ldots \ldots \ldots \ldots \ldots$} & 1998 May 23-24 & 5.527 & 4.946 & 4.812 & 0.007 & 0.002 & 0.002 & 6 \\
\hline & 1999 Jul 12-14 & 5.515 & 4.932 & 4.804 & 0.003 & 0.009 & 0.006 & 4 \\
\hline & Combined & 5.517 & 4.945 & 4.811 & 0.003 & 0.002 & 0.002 & 10 \\
\hline \multirow{3}{*}{ SA $107-347 \ldots \ldots \ldots \ldots \ldots$} & 1998 May 23-24 & 7.004 & 6.322 & 6.198 & 0.003 & 0.004 & 0.006 & 4 \\
\hline & 1999 Jul 12-14 & 7.025 & 6.338 & 6.207 & 0.016 & 0.008 & 0.008 & 4 \\
\hline & Combined & 7.005 & 6.325 & 6.201 & 0.003 & 0.004 & 0.005 & 8 \\
\hline \multirow[t]{3}{*}{ SA $107-484 \ldots \ldots \ldots \ldots \ldots$} & 1998 May 23-24 & 9.097 & 8.516 & 8.393 & 0.023 & 0.010 & 0.010 & 6 \\
\hline & 1999 Jul 12-14 & 9.133 & 8.509 & 8.392 & 0.047 & 0.012 & 0.024 & 5 \\
\hline & Combined & 9.104 & 8.513 & 8.393 & 0.021 & 0.008 & 0.009 & 11 \\
\hline \multirow[t]{3}{*}{ SA $108-475 \ldots \ldots \ldots \ldots \ldots$} & 1998 May 23-24 & 8.789 & 8.105 & 7.965 & 0.024 & 0.010 & 0.009 & 4 \\
\hline & 1999 Jul 12-14 & 8.761 & 8.108 & 7.942 & 0.023 & 0.027 & 0.020 & 5 \\
\hline & Combined & 8.774 & 8.105 & 7.961 & 0.017 & 0.009 & 0.008 & 9 \\
\hline \multirow[t]{3}{*}{ SA $108-827 \ldots \ldots \ldots \ldots \ldots$} & 1998 May 23-24 & 5.685 & 5.104 & 4.970 & 0.016 & 0.003 & 0.003 & 4 \\
\hline & 1999 Jul 12-14 & 5.679 & 5.092 & 4.958 & 0.005 & 0.009 & 0.006 & 4 \\
\hline & Combined & 5.680 & 5.103 & 4.968 & 0.005 & 0.003 & 0.003 & 8 \\
\hline \multirow[t]{3}{*}{ SA $108-1918 \ldots \ldots \ldots \ldots \ldots$} & 1998 May 23-24 & 8.816 & 8.147 & 8.000 & 0.022 & 0.007 & 0.006 & 4 \\
\hline & 1999 Jul 12-14 & 8.814 & 8.152 & 8.003 & 0.049 & 0.015 & 0.015 & 6 \\
\hline & Combined & 8.816 & 8.148 & 8.000 & 0.020 & 0.006 & 0.006 & 10 \\
\hline \multirow[t]{3}{*}{ SA $109-231 \ldots \ldots \ldots \ldots \ldots$} & 1998 May 23-24 & 6.659 & 6.031 & 5.866 & 0.020 & 0.012 & 0.011 & 4 \\
\hline & 1999 Jul 12-14 & 6.669 & 6.036 & 5.868 & 0.005 & 0.006 & 0.003 & 6 \\
\hline & Combined & 6.668 & 6.035 & 5.868 & 0.005 & 0.005 & 0.003 & 10 \\
\hline \multirow[t]{3}{*}{ SA $110-471 \ldots \ldots \ldots \ldots \ldots$} & 1998 May 23-24 & 4.918 & 4.236 & 4.07 & 0.006 & 0.011 & 0.005 & 4 \\
\hline & 1999 Jul 12-14 & 4.931 & 4.244 & 4.088 & 0.002 & 0.002 & 0.002 & 4 \\
\hline & Combined & 4.930 & 4.244 & 4.086 & 0.002 & 0.002 & 0.002 & 8 \\
\hline \multirow[t]{3}{*}{ SA $112-275 \ldots \ldots \ldots \ldots \ldots$} & 1998 May 23-24 & 7.751 & 7.164 & 7.047 & 0.005 & 0.003 & 0.005 & 2 \\
\hline & 1999 Jul 12-14 & 7.765 & 7.176 & 7.063 & 0.022 & 0.007 & 0.006 & 4 \\
\hline & Combined & 7.752 & 7.166 & 7.054 & 0.005 & 0.003 & 0.004 & 6 \\
\hline \multirow[t]{3}{*}{ SA $112-595 \ldots \ldots \ldots \ldots \ldots$} & 1998 May 23-24 & 8.287 & 7.470 & 7.280 & 0.006 & 0.008 & 0.007 & 4 \\
\hline & 1999 Jul 12-14 & 8.312 & 7.480 & 7.304 & 0.030 & 0.012 & 0.017 & 5 \\
\hline & Combined & 8.288 & 7.473 & 7.283 & 0.006 & 0.007 & 0.006 & 9 \\
\hline SA $113-259 \ldots \ldots \ldots \ldots \ldots$ & 1999 Jul 12-14 & 9.702 & 9.120 & 8.986 & 0.111 & 0.033 & 0.057 & 9 \\
\hline SA $113-269 \ldots \ldots \ldots \ldots \ldots$ & 1999 Jul 12-14 & 7.564 & 7.022 & 6.918 & 0.012 & 0.007 & 0.007 & 6 \\
\hline SA $114-176 \ldots \ldots \ldots \ldots \ldots$ & 1999 Jul 12-14 & 6.616 & 5.914 & 5.752 & 0.011 & 0.005 & 0.009 & 4 \\
\hline SA $114-548 \ldots \ldots \ldots \ldots \ldots$ & 1999 Jul 12-14 & 9.176 & 8.500 & 8.333 & 0.034 & 0.024 & 0.030 & 4 \\
\hline SA $114-656 \ldots \ldots \ldots \ldots \ldots$ & 1999 Jul 12-14 & 10.814 & 10.211 & 10.209 & 0.105 & 0.097 & 0.090 & 6 \\
\hline SA $114-670 \ldots \ldots \ldots \ldots \ldots$ & 1999 Jul 12-14 & 8.964 & 8.374 & 8.264 & 0.036 & 0.011 & 0.039 & 7 \\
\hline SA $115-427 \ldots \ldots \ldots \ldots \ldots$ & 1999 Jul 12-14 & 6.794 & 6.212 & 6.107 & 0.013 & 0.008 & 0.010 & 6 \\
\hline SA $115-516 \ldots \ldots \ldots \ldots \ldots$ & 1999 Jul 12-14 & 8.488 & 7.926 & 7.836 & 0.020 & 0.007 & 0.015 & 4 \\
\hline
\end{tabular}

the final version of the optically normalized supertemplate for this star (and for many others in this paper) will be constrained using photometry from Landolt, Menzies, Hipparcos, and Tycho.

TABLE 5

New Technique of Optical Normalization OF THE Supertemplate

\begin{tabular}{cccc}
\hline \hline Filter & Scale & $\begin{array}{c}\text { Fractional } \\
\text { Uncertainty }\end{array}$ & $\begin{array}{c}\lambda_{\text {iso }} \\
(\mu \mathrm{m})\end{array}$ \\
\hline Landolt $U \ldots \ldots \ldots \ldots \ldots$. & $3.995 \mathrm{E}-04$ & $5.360 \mathrm{E}-03$ & 0.3597 \\
Landolt $B \ldots \ldots \ldots \ldots \ldots$. & $6.029 \mathrm{E}-04$ & $5.190 \mathrm{E}-03$ & 0.4409 \\
Landolt $V \ldots \ldots \ldots \ldots \ldots$. & $5.988 \mathrm{E}-04$ & $4.853 \mathrm{E}-03$ & 0.5440 \\
Landolt $R \ldots \ldots \ldots \ldots \ldots$. & $6.158 \mathrm{E}-04$ & $4.660 \mathrm{E}-03$ & 0.6427 \\
Landolt $I \ldots \ldots \ldots \ldots \ldots \ldots$. & $5.333 \mathrm{E}-04$ & $4.827 \mathrm{E}-03$ & 0.8048 \\
Mean scale $\ldots \ldots \ldots \ldots .$. & $5.554 \mathrm{E}-04$ & $2.218 \mathrm{E}-03$ & $\ldots$ \\
\hline
\end{tabular}

Note.-An illustration for a Landolt K giant, SA 112-275, K0 III, $A_{V}=0.620$, using just Landolt's UBVRI photometry.
As Table 5 shows, the scale factor derived from the $U$ band data is obviously much smaller than those associated with the $B V R I$ bands. On detailed examination, we found this situation occurred for almost every star. Possible explanations for this kind of behavior are discussed by Bessell (1990, his $\S 6$ ). Therefore, because we wished these stars to be treated in the most precise way possible, we decided not to use the $U$ photometry for any of the sample of stars. Utilizing solely $B V R I$, but combining both Landolt's and Menzies' data, we obtained a scale factor of $(5.628 \pm 0.019) \times 10^{-4}, 1.3 \%$ larger than that in Table 5 . This difference is typical of the rest of our sample of stars.

\subsubsection{The Prediction of NIR Magnitudes}

To predict $J H K$, we integrated the Tenerife (TCS) NIR system response curves over the correctly scaled and reddened supertemplates and converted the NIR in-band fluxes into their corresponding TCS magnitudes. For assessment 
TABLE 6

Predicting Tenerife $J H K$ from the Final, Optically Normalized, Reddened SuPERTEMPLATE OF SA 112-275

\begin{tabular}{|c|c|c|c|c|c|}
\hline \multirow[b]{2}{*}{$\begin{array}{l}\text { FILTER } \\
\text { (1) }\end{array}$} & \multicolumn{2}{|c|}{ Predicted } & \multicolumn{2}{|c|}{ OBSERVED } & \multirow[b]{2}{*}{$\begin{array}{c}\text { DIFFERENCE } \\
\text { (6) }\end{array}$} \\
\hline & $\begin{array}{l}\text { Magnitude } \\
\text { (2) }\end{array}$ & $\begin{array}{c}\text { Uncertainty } \\
\text { (3) }\end{array}$ & $\begin{array}{l}\text { Magnitude } \\
\text { (4) }\end{array}$ & $\begin{array}{c}\text { Uncertainty } \\
\text { (5) }\end{array}$ & \\
\hline 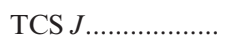 & 7.706 & 0.016 & 7.752 & 0.005 & +0.046 \\
\hline 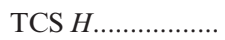 & 7.191 & 0.011 & 7.166 & 0.003 & -0.025 \\
\hline TCS $K \ldots \ldots \ldots \ldots \ldots$ & 7.083 & 0.010 & 7.054 & 0.004 & -0.029 \\
\hline MAD & $\ldots$ & $\ldots$ & $\ldots$ & $\ldots$ & -0.003 \\
\hline
\end{tabular}

of the accuracy of our predictions, we used the mean algebraic deviation (MAD) over the set of three TCS filters, in the sense observed minus predicted $J H K$. Ideally we would like to have the NIR photometry points "straddle" the normalized, reddened supertemplate, rather than to achieve the smallest mean absolute deviation for the three points, because that could arise by having a supertemplate lie entirely above, or below, the $J H K$ data, possibly implying an unsatisfactory bias. Accurate prediction of $J H K$ from a $B V R I$-constrained supertemplate or model depends on reliable estimates of spectral type, extinction, supertemplate or model shapes, and both optical and NIR photometry, making this a highly demanding process.

Table 6 shows the comparison of predicted and observed TCS $J H K$ magnitudes from the final optically constrained supertemplate for SA 112-275 (i.e., based on data from
Landolt, Menzies, Hipparcos, and Tycho; see the Appendix for a discussion of how the Tycho photometry was handled), from which the calculation of the MAD is performed. Column (1) shows the relevant TCS filter; columns (2) and (3) give the predicted magnitude and uncertainty after integrating the RSR for that filter over the supertemplate; columns (4) and (5) offer the corresponding observed magnitude and uncertainty; and column (6) lists the difference, column (4) minus column (2). The last row summarizes the MAD for the star as the unweighted mean of the numbers in column (6).

Table 7 summarizes these details for all $24 \mathrm{~K} / \mathrm{M}$ giants, with their supertemplate types, extinctions, final scale factors, biases, and MADs, incorporating both Landolt/TCS and Carter-Meadows/SAAO methods. For SA 108-475, both routes were used for our Mount Hopkins spectral class

TABLE 7

Cool Giant Supertemplates

\begin{tabular}{|c|c|c|c|c|c|c|}
\hline Star & Template & $A_{V}$ & Scale & $\begin{array}{l}\text { Bias } \\
(\%)\end{array}$ & MAD & $\begin{array}{c}\text { Photometry } \\
\text { Sources }\end{array}$ \\
\hline SA 92-336 ….............. & K0 III & 0.000 & $1.775 \mathrm{E}-03$ & 0.30 & +0.006 & Landolt/TCS \\
\hline SA 94-251 .................. & K1 III & 0.493 & $2.969 \mathrm{E}-05$ & 0.33 & -0.014 & Carter-Meadows/SAAO \\
\hline SA $103-526 \ldots \ldots \ldots \ldots \ldots$ & K0 III & 0.245 & $1.585 \mathrm{E}-04$ & 0.32 & -0.021 & Landolt/TCS \\
\hline SA $105-205 \ldots \ldots \ldots \ldots \ldots$ & K3 III & 0.353 & $2.063 \mathrm{E}-03$ & 0.32 & -0.028 & Landolt/TCS \\
\hline SA $105-405 \ldots \ldots \ldots \ldots \ldots$ & K5 III & 0.031 & $9.528 \mathrm{E}-04$ & 0.33 & -0.044 & Landolt/TCS \\
\hline SA 107-35 ……............. & K2 III & 0.263 & $3.943 \mathrm{E}-03$ & 0.29 & -0.029 & Landolt/TCS \\
\hline SA $107-347 \ldots$. & K1.5 III & 0.608 & $2.011 \mathrm{E}-04$ & 0.31 & -0.062 & Landolt/TCS \\
\hline SA $107-484 \ldots \ldots \ldots \ldots \ldots$ & K3 III & 0.000 & $1.445 \mathrm{E}-04$ & 0.38 & +0.018 & Landolt/TCS \\
\hline \multirow[t]{2}{*}{ SA $108-475 \ldots \ldots \ldots \ldots \ldots$} & K3 III & 0.406 & $2.110 \mathrm{E}-04$ & 0.39 & -0.042 & Landolt/TCS \\
\hline & & 0.434 & $2.156 \mathrm{E}-04$ & 0.92 & +0.008 & Carter-Meadows/SAAO \\
\hline SA $108-475 \ldots \ldots \ldots \ldots \ldots$ & & 0.422 & $2.143 \mathrm{E}-04$ & 0.33 & $\ldots$ & All optical plus NIR \\
\hline SA $108-827 \ldots \ldots \ldots \ldots \ldots$ & K2 III & 0.350 & $3.558 \mathrm{E}-03$ & 0.32 & +0.008 & Landolt/TCS \\
\hline SA $108-1918 \ldots \ldots \ldots \ldots . .$. & K3 III & 0.567 & $2.237 \mathrm{E}-04$ & 0.39 & +0.039 & Landolt/TCS \\
\hline SA 109-231 ................ & K2 III & 0.843 & $1.611 \mathrm{E}-03$ & 0.32 & -0.011 & Landolt/TCS \\
\hline 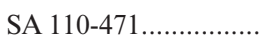 & K2 III & 0.803 & $8.465 \mathrm{E}-03$ & 0.31 & +0.036 & Landolt/TCS \\
\hline SA $112-275 \ldots \ldots \ldots \ldots \ldots$ & K0 III & 0.620 & $5.628 \mathrm{E}-04$ & 0.30 & -0.003 & Landolt/TCS \\
\hline SA $112-595 \ldots \ldots \ldots \ldots \ldots$ & M0 III & 0.174 & $2.098 \mathrm{E}-04$ & 0.35 & -0.030 & Landolt/TCS \\
\hline SA 113-259................. & K2 III & 0.012 & $8.157 \mathrm{E}-05$ & 0.31 & -0.008 & Landolt/TCS \\
\hline SA $113-269 \ldots \ldots \ldots \ldots \ldots$ & K0 III & 0.316 & $6.245 \mathrm{E}-04$ & 0.32 & +0.015 & Landolt/TCS \\
\hline SA $114-176 \ldots \ldots \ldots \ldots \ldots$ & K4 III & 0.046 & $3.388 \mathrm{E}-04$ & 0.29 & -0.014 & Landolt/TCS \\
\hline SA $114-548 \ldots \ldots \ldots \ldots \ldots$ & K3 III & 0.350 & $1.534 \mathrm{E}-04$ & 0.35 & +0.012 & Landolt/TCS \\
\hline SA $114-656 \ldots \ldots \ldots \ldots \ldots$ & K1 III & 0.000 & $5.062 \mathrm{E}-06$ & 0.43 & -0.016 & Landolt/TCS \\
\hline SA $114-670 \ldots \ldots \ldots \ldots \ldots$ & K1.5 III & 0.329 & $3.267 \mathrm{E}-04$ & 0.34 & +0.045 & Landolt/TCS \\
\hline SA $115-427 \ldots \ldots \ldots \ldots \ldots$ & K2 III & 0.000 & $1.159 \mathrm{E}-03$ & 0.30 & -0.025 & Landolt/TCS \\
\hline SA $115-516 \ldots \ldots \ldots \ldots \ldots$ & K1.5 III & 0.000 & $4.508 \mathrm{E}-05$ & 0.30 & +0.005 & Landolt/TCS \\
\hline 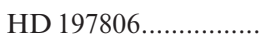 & K0 III & 0.530 & $6.526 \mathrm{E}-04$ & 0.53 & -0.013 & Carter-Meadows/SAAO \\
\hline
\end{tabular}

Note.-Listed are spectral types, $A_{V}$ 's, and final scale factors and biases, derived by normalizing using all available optical, NIR, and MIR photometry; near-infrared $(J H K)$ MADs are derived from the optical photometry listed in the final column. 
TABLE 8

Carter-Meadows A Dwarfs

\begin{tabular}{|c|c|c|c|c|c|c|}
\hline Star & Template & $A_{V}$ & Scale & $\begin{array}{l}\text { Bias } \\
(\%)\end{array}$ & MAD & $\begin{array}{c}\text { Photometry } \\
\text { Sources }\end{array}$ \\
\hline HD $15911 \ldots \ldots \ldots \ldots . . .$. & $\mathrm{A} 0 \mathrm{~V}$ & 0.000 & $1.272 \mathrm{E}+02$ & 1.14 & +0.005 & Carter-Meadows/SAAO \\
\hline 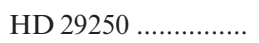 & $\mathrm{A} 4 \mathrm{~V}$ & 0.295 & $1.699 \mathrm{E}+02$ & 1.10 & +0.023 & Carter-Meadows/SAAO \\
\hline HD $62388 \ldots \ldots \ldots \ldots \ldots . . .$. & A0 $\mathrm{V}$ & 0.232 & $2.637 \mathrm{E}+02$ & 1.12 & -0.028 & Carter-Meadows/SAAO \\
\hline HD $71264 \ldots \ldots \ldots \ldots \ldots$ & A0 V & 0.406 & $3.152 \mathrm{E}+02$ & 1.16 & +0.029 & Carter-Meadows/SAAO \\
\hline 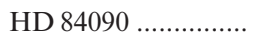 & $\mathrm{A} 3 \mathrm{~V}$ & 0.149 & $3.532 \mathrm{E}+02$ & 1.13 & +0.009 & Carter-Meadows/SAAO \\
\hline HD $105116 \ldots \ldots \ldots \ldots . . .$. & $\mathrm{A} 2 \mathrm{~V}$ & 0.372 & $5.138 \mathrm{E}+02$ & 1.12 & -0.053 & Carter-Meadows/SAAO \\
\hline HD $106807 \ldots \ldots \ldots \ldots . . . .$. & $\mathrm{A} 1 \mathrm{~V}$ & 0.276 & $2.850 \mathrm{E}+02$ & 1.09 & -0.035 & Carter-Meadows/SAAO \\
\hline HD $136879 \ldots \ldots \ldots \ldots \ldots . . . . . .$. & $\mathrm{A} 0 \mathrm{~V}$ & 0.521 & $3.124 \mathrm{E}+02$ & 1.13 & -0.006 & Carter-Meadows/SAAO \\
\hline HD $216009 \ldots \ldots \ldots \ldots . . . .$. & $\mathrm{A} 0 \mathrm{~V}$ & 0.155 & $5.171 \mathrm{E}+02$ & 1.08 & -0.008 & Carter-Meadows/SAAO \\
\hline
\end{tabular}

Note.-Listed are spectral types, $A_{V}$ 's, and final scale factors and biases, derived by normalizing using all available optical, NIR, and MIR photometry; near-infrared $(J H K)$ MADs are derived from the optical photometry listed in the final column.

of K3 III; that is, using Landolt $B V R I$ to predict Tenerife $J H K$, and using Carter-Meadows BVRI to predict CarterMeadows SAAO JHK. While the two derived extinctions are not identical, they are very close (0.028 mag apart), which may afford an independent method to assess the uncertainties in our derived values of $A_{V}$. We derived very similar scale factors and MADs, each pair differing only at the $1.6 \sigma$ level of the joint uncertainties. Consequently, the third record for this star represents the result of combining all its optical and NIR photometry and using an $A_{V}$ corresponding to the average of the two $B-V$ values.

The fact that the cool giants have biases (the percentage uncertainty in the mean scale factor for a supertemplate or model) that are all so homogeneous and well below $1 \%$ reflects primarily the precision achieved in both the Landolt $B V R I$ and TCS $J H K$ data sets, and secondarily the significant number of photometry points per star, between eight and 16 (many stars have data from both Landolt and Menzies, and some also have Hipparcos/Tycho-2 data). The larger values seen for stars constrained by SAAO photometry are essentially due to the more conservative uncertainties assigned by Carter to his $J H K$ magnitudes $( \pm 0.025)$ as compared with those from Tenerife (typically smaller by a factor of $\frac{1}{2}$ to $\frac{1}{3}$ ). With much sparser data sets, for example, just $B V J H K$, typical of some potential IRAC calibrators, supertemplate biases are larger, typically $\sim 2 \%-3 \%$.

Table 8 similarly presents our results on nine A dwarfs for which the types cited by Carter \& Meadows are confirmed by the Michigan Catalogue. The biases are all larger than those for the sample of cool giants because we assign an uncertainty of $\pm 5 \%$ to the synthetic spectra of A dwarfs, to account for the influence on the continuum and lines of deviations in line strengths, gravities, metallicities, and real stellar structures as compared with opacities and structures represented in models. Examination of the MADs in Tables 7 and 8 suggests the viability of predicting JHK from optically normalized, reddened supertemplates and models, chosen in accord with our Mount Hopkins spectral types for cool giants, and using types drawn from the Michigan Catalogue for A dwarfs. Indeed, the (unweighted) average of the MADs for the ensemble of 33 stars is $-0.007 \pm 0.005$.

Figure 6 presents the set of MADs for the A dwarfs and cool giants separately, broken down into the behavior at $J$,
$H$, and $K$. Every individual star is plotted in the appropriate diagrams with the $1 \sigma$ error bars on the values of the MAD. By and large, the sets of MADs are consistent with the ensembles of stars having essentially zero offset between observation and prediction at the $3 \sigma$ level, except perhaps for one or two stars.

\subsection{Predicting MIR Flux Densities \\ 3.6.1. IRAS}

For nine Landolt stars, we were also able to find IRAS measurements, either from the Point Source Catalog (PSC) or, more often, the Faint Source Catalog (FSC) or Faint Source Reject Catalog (FSR). These appear in Table 9, expressed as $F_{\nu}$ (in millijanskys). For these stars, using the same scale factors as implied by the optical normalizations, we predicted the 12 and $25 \mu \mathrm{m}$ flux densities using the IRAS system response curves, and these flux densities are also given in Table 9. In all cases, predictions lie within $\sim 1 \sigma$ of IRAS observations, further vindicating the efficacy of the technique used to create normalized supertemplates, and limiting the possibility that these cool giants might have circumstellar dust shells. None of the Carter-Meadows A dwarfs were detected by $I R A S$, indicating that, at a rather gross level, none of these stars are afflicted by the Vega phenomenon, in keeping with their intended usage as calibrators.

\subsection{2. $M S X$}

One star among the Landolt sample, SA 114-176, was observed and detected by $M S X$ at $8.28 \mu \mathrm{m}$. Table 10 summarizes its observed and predicted irradiances, isophotal flux densities, and magnitudes in this $M S X$ band, based on the scale factor for its supertemplate determined from optical normalization. All lie within $\sim 1.9 \sigma$ of the observed values, again validating our method. The final row in Table 10 shows the predicted $M S X$ quantities based on normalizing the supertemplate for this star using all optical and NIR data. There is almost no difference between the predictions determined from optical data and those from from opticalplus-NIR data, essentially because the small MAD for this star (Table 7) indicates that the two mean scale factors must be very similar. 

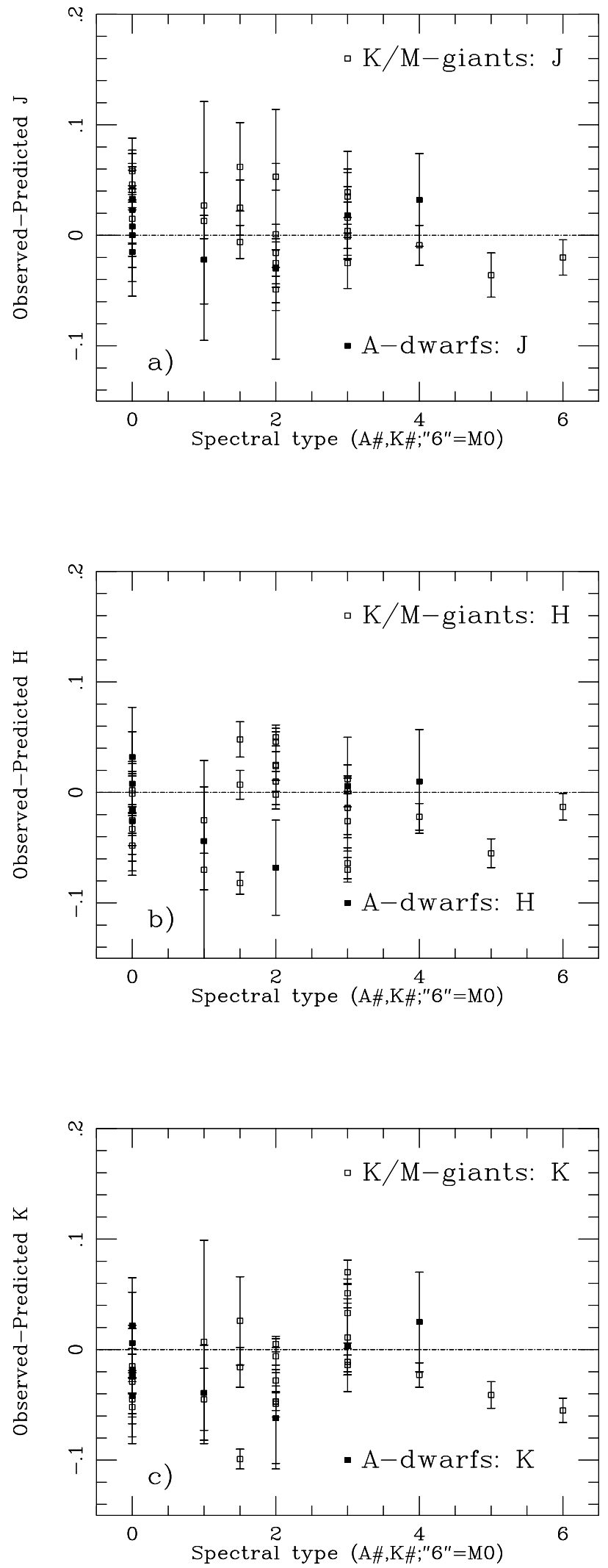

FIG. 6.-The individual stars of our test sample with their MADs and $1 \sigma$ uncertainties. Both A dwarfs and cool giants are plotted together at each of the three wavelengths: (a) $J$; (b) $H$; (c) $K$.

\section{SUPERTEMPLATE FILES AND HOW TO OBTAIN THEM}

Having demonstrated the NIR predictability of these Landolt and Carter-Meadows stars from the spectral types, supertemplates or models, and optical photometry, we have renormalized each spectrum by utilizing every available optical, NIR, and MIR photometric point to define the mean scale factor and bias for each templated star. These final spectra elevate our subset of the precision optical standards into IR calibrators and are self-consistent with our published set of almost 450 fiducial IR standards. As an illustration of how the spectra are constrained by the photometry, Figure 7 shows supertemplates or models for eight of the 33 stars.

The 33 supertemplates/models are available through the electronic version of this paper. A detailed header precedes every supertemplate and model created as we have described. This header is accompanied by various names for each star; release version, date, and time of spectrum creation; the supertemplate spectrum used; the extinctions both of the bright star that gave rise to the supertemplate shape and of the templated star; for cool giants, the angular diameter deduced for the stellar supertemplate (derived from the template scale factor and our published values of angular diameters for the bright composite spectra; Cohen et al. 1996b); all the characterized photometry used to normalize the supertemplate, with references; and our determinations, for each filter used, of isophotal flux and its uncertainty, and isophotal wavelength for the particular spectrum in question. Supertemplates are always named for their HD designations, when a star has an HD number, for example, "HD139513.tem" (for the star SA 107-347); otherwise, the Selected Area star name will represent the file, for example, "SA113_259.tem."

The actual calibrated stellar spectra have a five-column format (exactly as in Paper X). For the wavelength range from 0.275 to $35.00 \mu \mathrm{m}$, we tabulate wavelength $(\mu \mathrm{m})$, monochromatic irradiance $\left(F_{\lambda}\right.$ in units of $\left.\mathrm{W} \mathrm{cm}{ }^{-2} \mu \mathrm{m}^{-1}\right)$, total uncertainty (also in units of $\mathrm{W} \mathrm{cm}^{-2} \mu \mathrm{m}^{-1}$ ) associated with this value of $F_{\lambda}$, local bias, and global bias. For most applications, "total uncertainty" is the error term most appropriate to use. It is the standard deviation of the spectral irradiance and incorporates the local and global biases. Local and global biases are given as percentages of the irradiance. The global bias does not contribute error to flux ratios or color measurements and may be removed (in quadrature) from the total error. Note that we prefer to provide pristine data rather than to regrid each supertemplate to an equally spaced or common wavelength scale. Each supertemplate has a different set of wavelengths in the IR. Consequently, spectra are not tabulated at equal intervals of wavelength but follow Pickles (1998) as far as $2.500 \mu \mathrm{m}$ and then mimic the wavelengths of the originally observed composite spectra.

In the electronic version of this paper, the star names in the first column of Table 7 are links to the supertemplates for the 24 Landolt stars (only the last entry for SA 108-475 has such a link), while Table 8 similarly links to the supertemplates for the nine Carter-Meadows A dwarfs.

Should other well-characterized optical or IR observations of any of these 33 stars become available at some time in the future that are not directly calibrated by an earlier version of the same star's spectrum, it would be possible to 


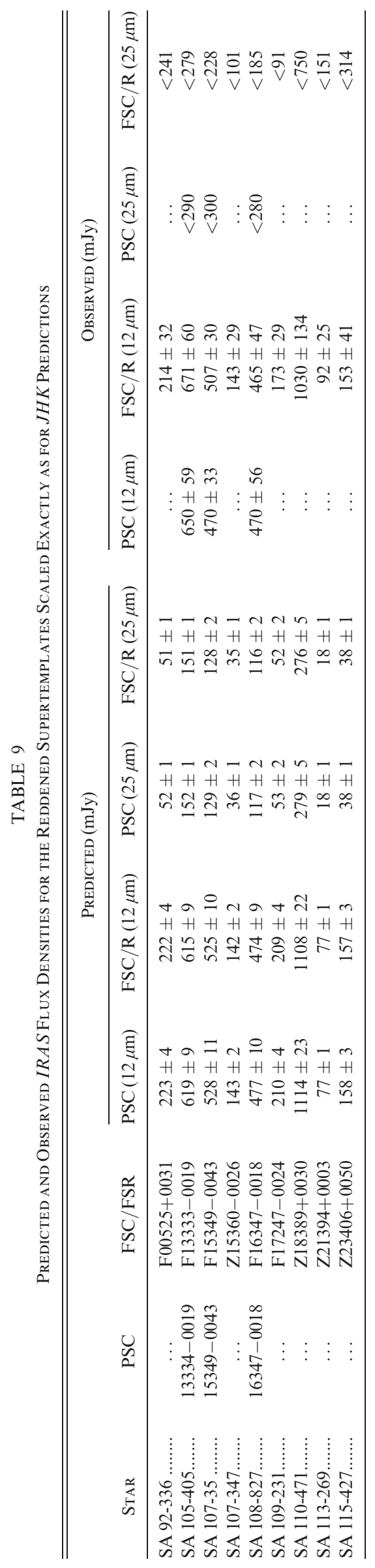


TABLE 10

$M S X 8.28 \mu \mathrm{m}$ ATtRIBUtes FOR SA 114-176

\begin{tabular}{|c|c|c|c|c|}
\hline Photometry & $\begin{array}{c}\text { Irradiance } \\
\left(10^{-18} \mathrm{~W} \mathrm{~cm}^{-2} \mu \mathrm{m}^{-1}\right)\end{array}$ & $\begin{array}{c}F_{\lambda} \text { (iso) } \\
\left(10^{-18} \mathrm{~W} \mathrm{~cm}^{-2} \mu \mathrm{m}^{-1}\right)\end{array}$ & $\begin{array}{l}{[8.28]} \\
(\mathrm{mag})\end{array}$ & $\begin{array}{c}F_{\nu} \\
(\mathrm{mJy})\end{array}$ \\
\hline Observed $\ldots \ldots \ldots \ldots \ldots \ldots \ldots \ldots$ & $3.81 \pm 0.24$ & $1.13 \pm 0.07$ & $5.83 \pm 0.07$ & $272 \pm 17$ \\
\hline Predicted (No. 1) .............. & $4.43 \pm 0.22$ & $1.32 \pm 0.09$ & $5.67 \pm 0.06$ & $316 \pm 16$ \\
\hline Predicted (No. 2) ............... & $4.43 \pm 0.22$ & $1.32 \pm 0.09$ & $5.67 \pm 0.06$ & $316 \pm 16$ \\
\hline
\end{tabular}

NoтE.-Summary for the reddened supertemplate of SA 114-176 scaled exactly as for $J H K$ predictions (No. 1) and again using all of $B V R I B_{\mathrm{T}} V_{\mathrm{T}} J H K($ No. 2).

recreate that supertemplate or model, hopefully with reduced uncertainties. This procedure was followed when $M S X$ measurements first became available for a number of $\mathrm{K} / \mathrm{M}$ giants from Paper $\mathrm{X}$, resulting in the issuance of updated spectra, with reduced template biases (Walker \& Cohen 2002). The headers of the associated stellar spectra always carry the date of creation of the updated products, so there will be no confusion as to which generation of calibration spectrum is represented when these files are downloaded.

\section{CONCLUSIONS}

The purpose of this paper has been threefold: to demonstrate the efficacy of our approach to SIRTF/IRAC calibrators; to validate the resulting scale factors, spectral types, and extinctions using different optical data sets for a new, faint set of IR calibrators; and to provide an archive of absolutely calibrated optical-to-infrared supertemplates and models for stars between 2 and 3 orders of magnitude fainter than our current published network of IR standards. We have been able to derive mean scale factors for a set of cool giant supertemplates and hot dwarf models, drawn from a set of much fainter potential calibration stars than we have previously established, with supertemplate and model normalizations based solely on either ground-based or space-based optical photometry. We have demonstrated the capability of closely predicting the NIR (and in some cases even MIR) brightnesses of these stars using appropriately reddened supertemplates or models based on a modern set of MK spectral classifications for these potential calibrators. When applied to the actual calibrators for SIRTF, the combination of NIR and MIR points helps to reduce both random and systematic uncertainties in the $3-10 \mu \mathrm{m}$ range covered by IRAC.

For the present, we have offered a method that can successfully predict NIR $J H K$ magnitudes for a sample of stars of well-determined spectral type in the ranges $\mathrm{K} 0-\mathrm{M} 0$ III and A0-A5 V. Therefore, combining optical and NIR photometry (with MIR when available) on such stars and renormalizing their supertemplates should satisfy our goal of having a set of absolutely calibrated optical-IR spectral energy distributions that will meet the uncertainties required for IRAC calibrators. We have attempted to quantify the uncertainties we expect for potential calibrators by predicting the four IRAC magnitudes and isophotal flux densities $\left(F_{\lambda}, F_{\nu}\right)$, and their uncertainties, for the faintest $\mathrm{A}$ dwarf (HD 15911, A0 V, $K \approx 9.5$ ) and K giant (SA 114-656, K1 III, $K \approx 10.2$ ) in our sample of 33 stars. Table 11 presents this information by band for each star. The final column in the table offers the absolute error in the in-band flux expressed in terms of the percentage fractional uncertainty. These absolute errors include the estimated uncertainties in the IRAC relative spectral response curves, in our supertemplates and Kurucz models, in the extinction law used, and in the normalizing photometry. All errors are well within the total error budget of $10 \%$ absolute set for IRAC. For the cool giant stars, molecular absorption bands contribute to IRAC2 and IRAC3 (the CO fundamental) and IRAC4 (the $\mathrm{SiO}$ fundamental), driving up the empirical errors in these features in the observations of the original composites from which supertemplates were derived. We estimate that significantly fainter stars with poor and sparse photometry could have uncertainties of $\sim 5 \%$ in the IRAC bands. However, even these errors leave a significant component of the budget to instrumental phenomena, such as the characterization of any nonlinearities, and timedependent responsivities on-orbit.

In reality, although this work was undertaken to provide direct calibrational support for IRAC, there are additional constraints on the eventual SIRTF calibrators, namely, that they should lie in the constant-viewing zones surrounding each ecliptic pole. None of the Selected Areas lie near the ecliptic poles. However, an additional solution we have

TABLE 11

EXPeCted IRAC Attributes For the Two Faintest Stars in OUR Sample of Landolt/Carter-Meadows ObJects

\begin{tabular}{|c|c|c|c|c|c|c|c|c|c|}
\hline Star & Type & Band & Mag. & $\begin{array}{l}\text { Mag. } \\
\text { Unc. }\end{array}$ & $\begin{array}{c}F_{\lambda} \\
\left(\mathrm{W} \mathrm{cm}^{-2} \mu \mathrm{m}^{-1}\right)\end{array}$ & $\begin{array}{c}F_{\lambda} \text { Unc. } \\
\left(\mathrm{W} \mathrm{cm}^{-2} \mu \mathrm{m}^{-1}\right)\end{array}$ & $\begin{array}{l}F_{\nu} \text { (iso) } \\
(\mathrm{mJy})\end{array}$ & $\begin{array}{l}F_{\nu} \text { (iso) Unc. } \\
\quad(\mathrm{mJy})\end{array}$ & $\begin{array}{c}\text { Frac. Unc. } \\
\qquad(\%)\end{array}$ \\
\hline \multirow[t]{4}{*}{ HD $15911 \ldots \ldots \ldots \ldots . . . . .}$. & \multirow[t]{4}{*}{$\mathrm{A} 0 \mathrm{~V}$} & IRAC1 & 9.470 & 0.023 & $1.07 \mathrm{E}-18$ & $2.08 \mathrm{E}-20$ & 45.1 & 0.88 & 1.94 \\
\hline & & IRAC2 & 9.470 & 0.023 & $4.32 \mathrm{E}-19$ & $8.38 \mathrm{E}-21$ & 29.2 & 0.57 & 1.94 \\
\hline & & IRAC3 & 9.469 & 0.023 & $1.77 \mathrm{E}-19$ & $3.42 \mathrm{E}-21$ & 19.0 & 0.37 & 1.94 \\
\hline & & IRAC4 & 9.472 & 0.023 & $4.90 \mathrm{E}-20$ & $9.46 \mathrm{E}-22$ & 10.2 & 0.20 & 1.93 \\
\hline \multirow{4}{*}{ SA $114-656 \ldots \ldots \ldots \ldots . . . .}$. & \multirow[t]{4}{*}{ K1 III } & IRAC1 & 10.045 & 0.023 & $6.10 \mathrm{E}-19$ & $1.28 \mathrm{E}-20$ & 25.7 & 0.54 & 2.10 \\
\hline & & IRAC2 & 10.155 & 0.030 & $2.22 \mathrm{E}-19$ & $6.19 \mathrm{E}-21$ & 15.0 & 0.42 & 2.78 \\
\hline & & IRAC3 & 10.117 & 0.018 & $9.41 \mathrm{E}-20$ & $1.56 \mathrm{E}-21$ & 10.1 & 0.17 & 1.66 \\
\hline & & IRAC4 & 10.063 & 0.019 & $2.75 \mathrm{E}-20$ & $4.96 \mathrm{E}-22$ & 5.75 & 0.10 & 1.80 \\
\hline
\end{tabular}



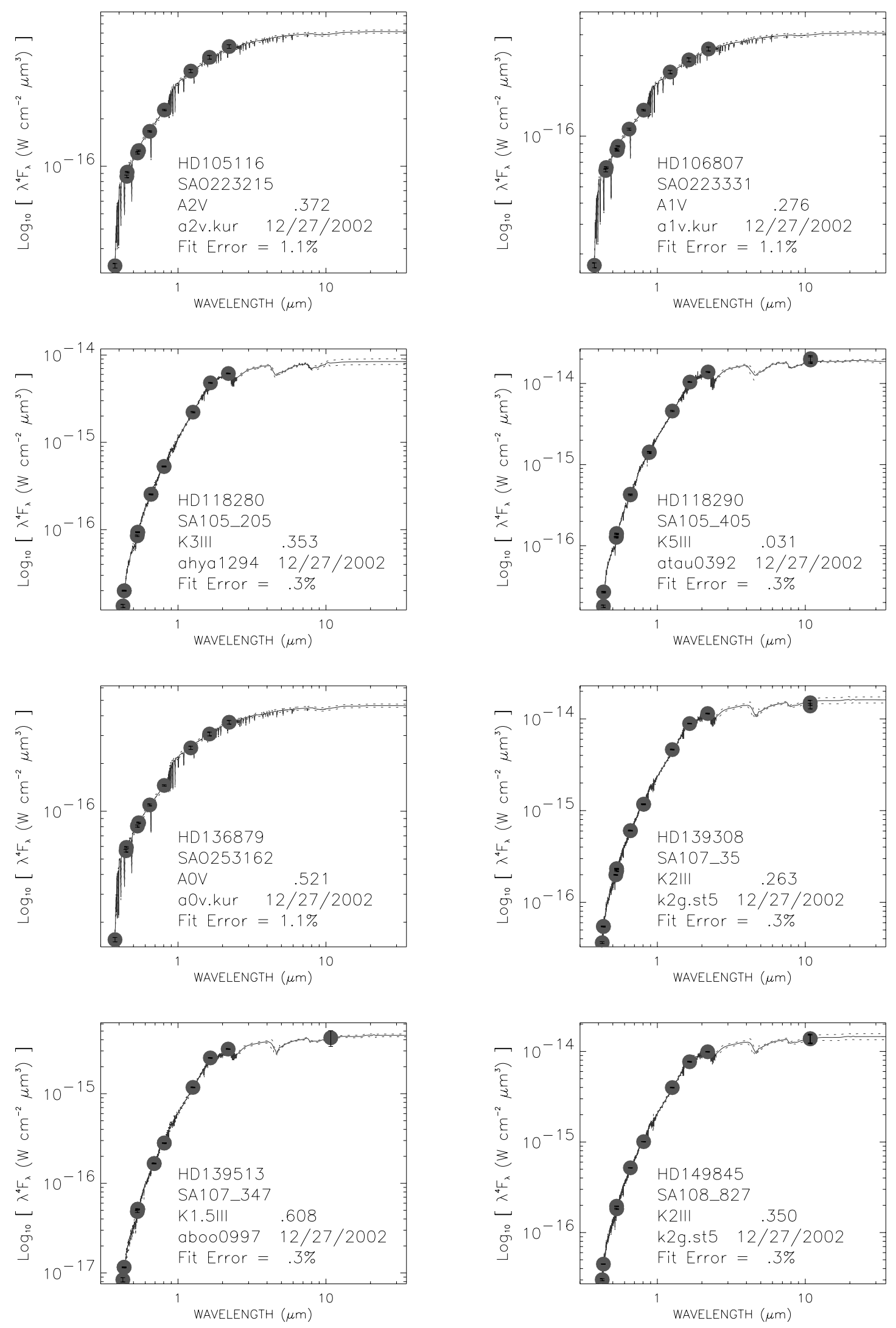

FIG. 7.-Montage of eight supertemplates and models drawn from the 24 cool giants and nine hot dwarfs discussed in this paper. Mean curves (solid lines) are flanked by $\pm 1 \sigma$ uncertainty bounds (dashed lines). The normalizing photometry is represented by circles with $\pm 1 \sigma$ error bars (sometimes within the circle). 
proposed to meet IRAC's calibration needs is a subset of stars distributed in longitude around the ecliptic plane, within $10^{\circ}$ of this plane in ecliptic latitude. This extra set of standards would support calibration either immediately prior to or following data downlinks, when SIRTF will lie in, or close to, the ecliptic plane. Some Selected Area stars, of relevant spectral type and with Landolt photometry, lie within this ecliptic plane zone. Details of the ecliptic pole and plane stars actually selected to support IRAC, and the derivation of their absolute spectra, will appear in a later paper in this series.

From the supertemplates, one can derive the expected attributes of the stars within any user-specified set of passbands accommodated by the range of our spectra, given the system's relative spectral response curves, and one can utilize the absolute spectra directly to support the calibration of low-resolution (resolving power $\sim 100$ ) spectrometers, exactly as for the 422 absolute template spectra published in Paper X. Thus, the supertemplate technique offers the capability to predict IRAC magnitudes and to support lowresolution operations with SIRTF's Infrared Spectrometer, thereby providing potential "cross calibrators" for this pair of instruments.

The acquisition of ground-based characterized $J H K$ photometry can be laborious, particularly when one deals with potential calibration stars significantly fainter than those discussed in the present paper, and some of SIRTF's calibrators lie in the southern hemisphere. Therefore, we have made further use of these 33 stars of intermediate brightness, whose optical spectra are proved to be good predictors of NIR magnitudes, to extend our absolute calibration framework to embrace 2MASS's $J H K_{s}$ bands (Cohen et al. 2003). Thereafter, 2 MASS photometry will provide an important resource for the assembly of even fainter calibrators for SIRTF.

M. C.'s work on IRAC's calibrators is supported by contract SV9-69008 between the University of California, Berkeley and the Smithsonian Astrophysical Observatory. We are grateful to Arlo Landolt for supplying electronically readable tables of his network of stars, and of his filters and phototube response curves, and to Andrew Pickles for his extremely helpful and detailed review. We thank David Shupe for extracting the most accurate coordinates from SIMBAD for the Landolt stars for our first observing runs at Mount Hopkins, and Mike Calkins and Perry Berlind for their invaluable efforts to secure FAST spectra. We thank Fred Witteborn, Jesse Bregman, and Diane Wooden for the use of the unpublished HIFOGS KAO spectra of $\beta$ UMi from one of our collaborative flight series in 1995. This research has made use of the SIMBAD database, operated at CDS, Strasbourg, France.

\section{APPENDIX}

\section{SPACE-BASED VISIBLE PHOTOMETRY}

As a further test of the supertemplate method, and because we may well encounter potential IRAC calibration stars that lack any characterized $U B V$ photometry, we have compared independent normalizations of supertemplates solely from space-based optical photometry (Tycho $B_{\mathrm{T}}$ and $V_{\mathrm{T}}$ [Høg et al. 1997] and $H_{p}$ [from Hipparcos, when available]) against benchmark normalizations using solely Landolt's $B V R I$ data for stars in the celestial equator (to which the published Landolt network is confined). Bessell (2000) has discussed the problems and inadequacy of the published information that accompanies the system response curves of the two Tycho bands $\left(B_{\mathrm{T}}, V_{\mathrm{T}}\right)$ and the single Hipparcos band $\left(H_{p}\right)$ given in Volume 1 of the Hipparcos archival volumes (ESA 1997). We adopted Bessell's (2000) recommended relative spectral response curves, which permit meaningful synthetic photometry. Following Bessell et al. (1998) and by interpolation, we adopted space-based optical magnitudes for Vega of $B_{\mathrm{T}}=0.028, V_{\mathrm{T}}=0.030$, and $H_{p}=0.029$. Table 12 contains the zero-magnitude attributes for the Hipparcos and Tycho bands, in the same manner as for the $U B V R I$ calibrations in Table 3.

We compared mean scale factors (applied to supertemplates to match predicted and observed irradiances) and their absolute uncertainties for cool giants, for 12 Landolt stars, and for one Carter-Meadows giant, represented by supertemplates that are normalized by ground-based and by space-based optical photometry. The scale factors derived for these giants from the space-based optical data are systematically a few percent smaller than those from ground-based BVRI. To investigate this phenomenon in greater depth, we have examined the individual scale factors derived from the $B_{\mathrm{T}}, V_{\mathrm{T}}$, and $H_{p}$ bands. Invariably, the $B_{\mathrm{T}}$ scale is less than the $V_{\mathrm{T}}$ scale, while the $V_{\mathrm{T}}$ scale is essentially equal to that found from $H_{p}$. We note that this phenomenon is not seen for the Carter-Meadows A dwarfs, for which the Hipparcos/Tycho bands available yield practically identical scale factors. Further analysis reveals the fact that, even for the cool giants, only the factors derived from $B_{\mathrm{T}}$ are in question. We note that $B_{\mathrm{T}}$ is strongly concentrated to the region around $4000 \AA$, where cool giant spectra are falling very steeply, below the $\mathrm{Ca} \mathrm{H}$ and $\mathrm{K}$ break, rendering these stars particularly faint in $B_{\mathrm{T}}$.

Therefore, we decided to investigate possible nonlinearities in the $B_{\mathrm{T}}$ scale at faint levels by plotting the ratios of $B V R I$ scale factors to those from the three space-based bands against Hipparcos/Tycho magnitude, for all our Landolt cool giants and our Carter-Meadows A dwarfs. Figures 8, 9, and 10 offer these plots for $B_{\mathrm{T}}, V_{\mathrm{T}}$, and $H_{p}$,

TABLE 12

Absolute Calibration of the Hipparcos-Tycho Optical Photometry Systems supporting this work

\begin{tabular}{|c|c|c|c|c|c|c|c|}
\hline Filter & $\begin{array}{l}\text { In-Band Flux } \\
\left(\mathrm{W} \mathrm{cm}^{-2}\right)\end{array}$ & $\begin{array}{l}\text { In-Band Uncertainty } \\
\qquad\left(\mathrm{W} \mathrm{cm}^{-2}\right)\end{array}$ & $\begin{array}{l}\text { Bandwidth } \\
\qquad(\mu \mathrm{m})\end{array}$ & $\begin{array}{c}F_{\lambda} \text { (iso) } \\
\left(\mathrm{W} \mathrm{cm}^{-2} \mu \mathrm{m}^{-1}\right)\end{array}$ & $\begin{array}{l}\lambda(\text { iso }) \\
(\mu \mathrm{m})\end{array}$ & $\begin{array}{c}F_{\nu}(\text { iso }) \\
(\mathrm{Jy})\end{array}$ & $\begin{array}{l}\mathrm{AB}_{\nu} \\
(\mathrm{mag})\end{array}$ \\
\hline Tycho $B_{\mathrm{T}} \ldots \ldots \ldots \ldots \ldots \ldots$ & $4.595 \mathrm{E}-13$ & $8.656 \mathrm{E}-15$ & 0.0685 & $6.714 \mathrm{E}-12$ & 0.4394 & 3943 & -0.090 \\
\hline 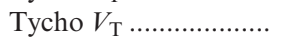 & $4.156 \mathrm{E}-13$ & $8.669 \mathrm{E}-15$ & 0.1033 & $4.025 \mathrm{E}-12$ & 0.5323 & 3761 & -0.038 \\
\hline Hipparcos $H_{p} \ldots \ldots \ldots \ldots \ldots$ & $9.412 \mathrm{E}-13$ & $1.941 \mathrm{E}-14$ & 0.2383 & $3.950 \mathrm{E}-12$ & 0.5355 & 3748 & -0.034 \\
\hline
\end{tabular}

NoтE.-Quantities tabulated correspond to the definition of zero magnitude in each filter. 


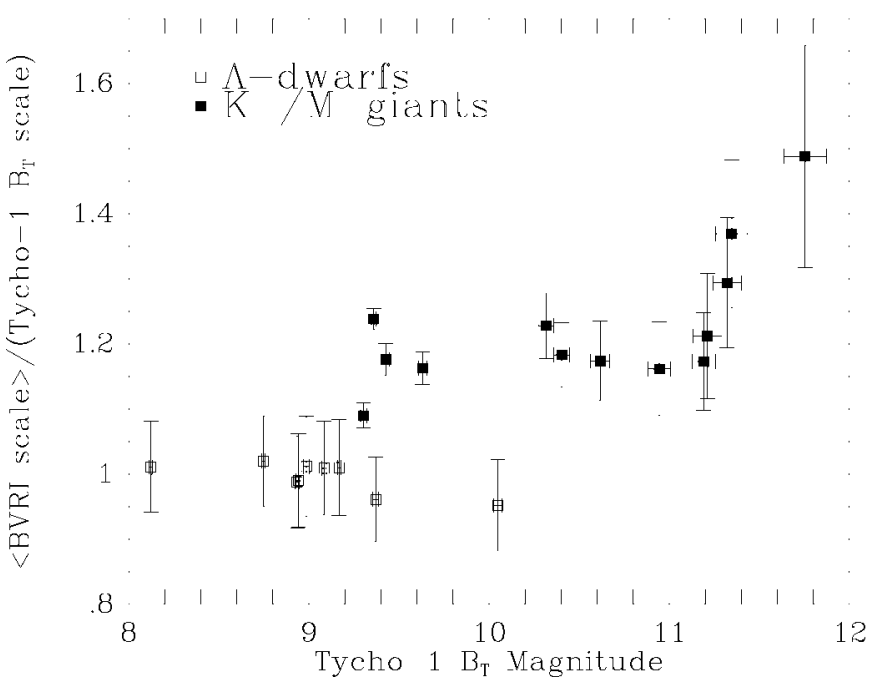

FIG. 8.- Ratio of ground-based to space-based optical photometric normalization factors for cool giants (filled squares) and A dwarfs (open squares) against Tycho-1's $B_{\mathrm{T}}$ magnitude.

respectively, distinguishing between $\mathrm{K} / \mathrm{M}$ giants and $\mathrm{A}$ dwarfs. On inspection, the A stars never show any dependence of the ratio of scales on brightness, even in $B_{\mathrm{T}}$, while the $\mathrm{K} / \mathrm{M}$ stars present an obvious trend toward larger ratios at fainter magnitudes in $B_{\mathrm{T}}$ but no similar trends in the other two bands.

These trends are quantified in Table 13, where we give the inverse-variance-weighted mean ratios of ground-based to Hipparcos/Tycho scale factors for all the stars in Tables 7 and 8 . Table 14 presents the slopes of the regression lines of scale ratio against space-based magnitude. The concurrence of these three ways of examining the data on normalization ratios points to the conclusion that for the faintest cool giants, one should not rely on the normalization factor derived directly from the $B_{\mathrm{T}}$ band. If a faint cool giant has $B V R I$ and space-based data, then the influence of $B_{\mathrm{T}}$ on the overall scale factor will be limited because the associated

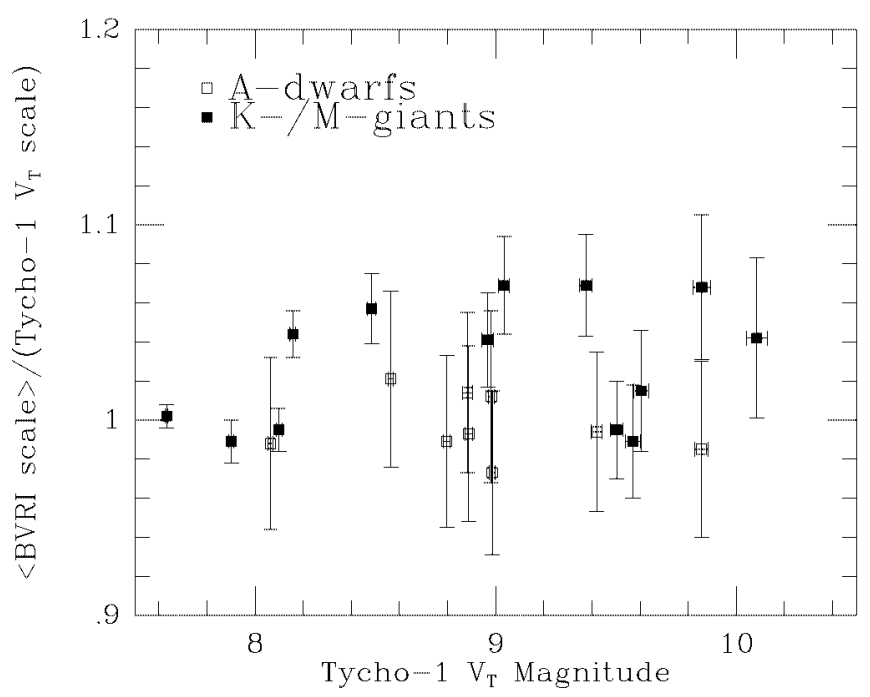

Fig. 9.- Same as Fig. 8, but for Tycho-1's $V_{\mathrm{T}}$ magnitude

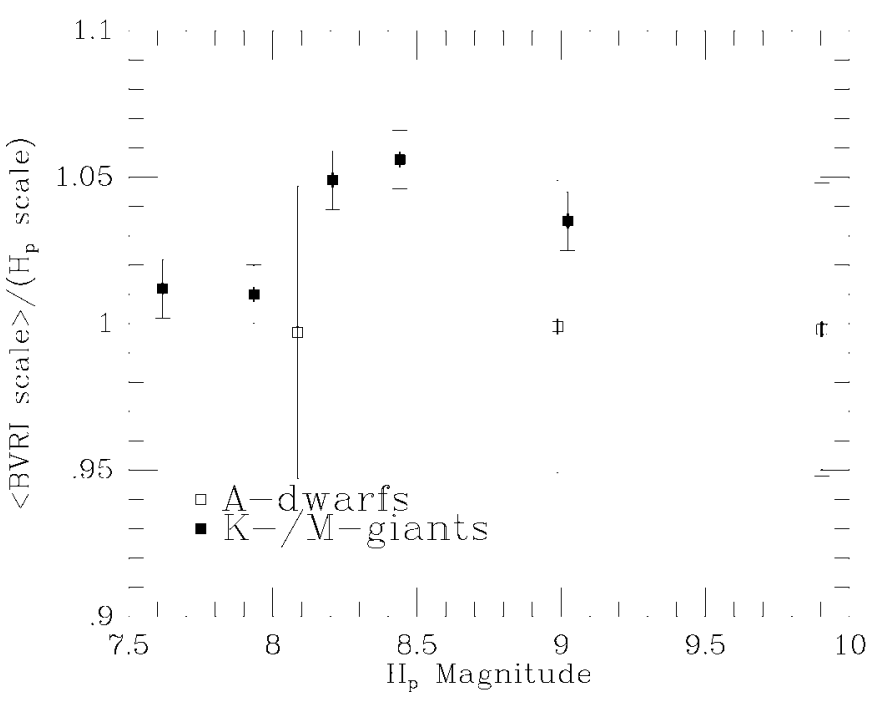

FIG. 10.- Same as Fig. 8, but for $H_{p}$

uncertainty in a faint $B_{\mathrm{T}}$ magnitude will be large enough that the weight accorded to $B_{\mathrm{T}}$ 's contribution under inversevariance weighting will be correspondingly small. If a cool giant lacks any $U B V(R I)$ photometry but Hipparcos/Tycho measurements exist, one could in principle ignore $B_{\mathrm{T}}$ 's scale factor. However, this could reduce the number of optical normalizing wavelengths to only a single, $V_{\mathrm{T}}$, point or, at best, two points, if $H_{p}$ is also available. To handle such situations, we suggest that it is important to use the regression line to increase the $B_{\mathrm{T}}$ scale factor, in accord with this line, so that it would mimic the scale that would have been obtained using $B V R I$ observations.

The fact that A dwarfs exhibit no such anomalies even in this bluest Hipparcos/Tycho band argues for the effect to arise from the extremely different energy distributions of the $\mathrm{K} / \mathrm{M}$ giants, whose faintest optical region lies within $B_{\mathrm{T}}$, and the A stars, which are brightest in the blue. A simple test of this conjecture would be to seek a dependence of the $B_{\mathrm{T}}$ scale factor on spectral type. The set of spectral classes probed is $\mathrm{K} 0, \mathrm{~K} 1.5, \mathrm{~K} 2, \mathrm{~K} 3, \mathrm{~K} 4$, and $\mathrm{K} 5 \mathrm{III}$, represented in our data set by $4,1,5,1,1$, and 1 star, respectively. A linear regression analysis using inverse-variance weighting for the scale factors found at each spectral class indicates an offset of $1.12 \pm 0.02$ (so that, even at type K0 III, the $B_{\mathrm{T}}$ scale is not unity) and a significant slope of $0.04 \pm 0.01$. Figure 11 displays these data and the formal best-fit regression line.

Since the time we began this program, the Tycho-2 Catalogue has appeared (Høg et al. 2000). This is substantially

TABLE 13

Ground-Based Supertemplate Normalization Compared With Hipparcos/Tycho-1 Scale Factors

\begin{tabular}{|c|c|c|c|}
\hline Filter & $\mathrm{K} / \mathrm{M}$ Giants & A Dwarfs & All Stars \\
\hline & $1.180 \pm 0.009$ & $0.994 \pm 0.024$ & $1.155 \pm 0.009$ \\
\hline$V_{\mathrm{T}} \ldots \ldots \ldots \ldots \ldots \ldots$ & $1.012 \pm 0.004$ & $0.997 \pm 0.014$ & $1.011 \pm 0.004$ \\
\hline$H_{p} \ldots \ldots \ldots \ldots \ldots \ldots$ & $1.032 \pm 0.011$ & $0.998 \pm 0.029$ & $1.030 \pm 0.011$ \\
\hline
\end{tabular}

Note.-Comparison as a function of space-based brightness: inverse-variance-weighted mean ratios and $\sigma$ 's of scale factors 
TABLE 14

Slopes of the Regression Lines of Scale Factors against Space-based Magnitudes

\begin{tabular}{cccc}
\hline \hline Filter & K/M Giants & A Dwarfs & All Stars \\
\hline Tycho-1: & & & \\
$B_{\mathrm{T}} \ldots \ldots .$. & $+0.033 \pm 0.018$ & $-0.036 \pm 0.052$ & $+0.062 \pm 0.016$ \\
$V_{\mathrm{T} \ldots \ldots .}$ & $+0.020 \pm 0.006$ & $-0.007 \pm 0.025$ & $+0.015 \pm 0.006$ \\
$H_{p} \ldots \ldots$. & $+0.061 \pm 0.026$ & $+0.001 \pm 0.031$ & $+0.037 \pm 0.026$ \\
Tycho-2: & & & \\
$B_{\mathrm{T}} \ldots \ldots .$. & $+0.018 \pm 0.019$ & $-0.028 \pm 0.048$ & $+0.034 \pm 0.015$ \\
$V_{\mathrm{T}} \ldots \ldots$. & $+0.015 \pm 0.010$ & $-0.008 \pm 0.026$ & $+0.011 \pm 0.010$ \\
$H_{p} \ldots \ldots$. & $+0.015 \pm 0.019$ & $-0.003 \pm 0.029$ & $+0.015 \pm 0.024$ \\
\hline
\end{tabular}

NOTE.-Slopes of the (ground-based or space-based) regression lines are in the three Hipparcos/Tycho bands, based on Tycho-1 and Tycho-2 photometry.

larger than Tycho-1 (2.5 million stars as compared with 1 million) and has higher quality photometry. Therefore, we decided to examine data from Tycho-2 in light of the trends found in Tycho-1 described above. Figures 12 and 13 present the identical information to Figures 8 and 9, but based on Tycho- 2 magnitudes. This time the ratios of supertemplate scale factors for the cool giants do not show any significant slope. The formal slopes and offsets of the regression lines against $B_{\mathrm{T}}$ are $0.02 \pm 0.02$ and $0.96 \pm 0.20$ (for $\mathrm{K}$ giants) and $-0.03 \pm 0.05$ and $1.27 \pm 0.44$ (A dwarfs). Therefore, we have replaced our Tycho-1 photometry for the stars in this paper with the corresponding data from Tycho-2 whenever available. For 12 cool giants with both Tycho-1 and Tycho- 2 photometry, we have investigated the effects of making this change on the ratios of $B V R I$ determined scale factors to those from Tycho alone. In $B_{\mathrm{T}}$, these ratios are worse (i.e., larger) with Tycho-2 than with Tycho-1 for three stars, unchanged for two stars, and better for seven. In $V_{\mathrm{T}}$, where the effects are much smaller in magnitude, the ratios are worse for two stars, unchanged for five, and better for five. The regression analysis for Tycho-2

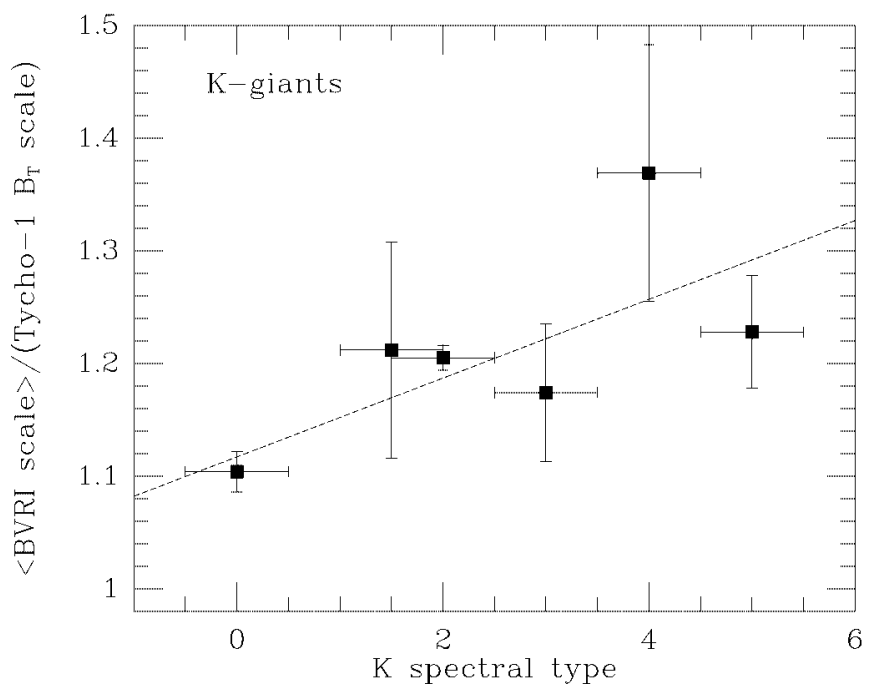

FIG. 11.- Ratio of ground-based to Tycho- $1 B_{\mathrm{T}}$ photometric normalization factors for $\mathrm{K}$ giants against $\mathrm{K}$ spectral class. The dashed line represents the formal regression line, and the slope is statistically significant.

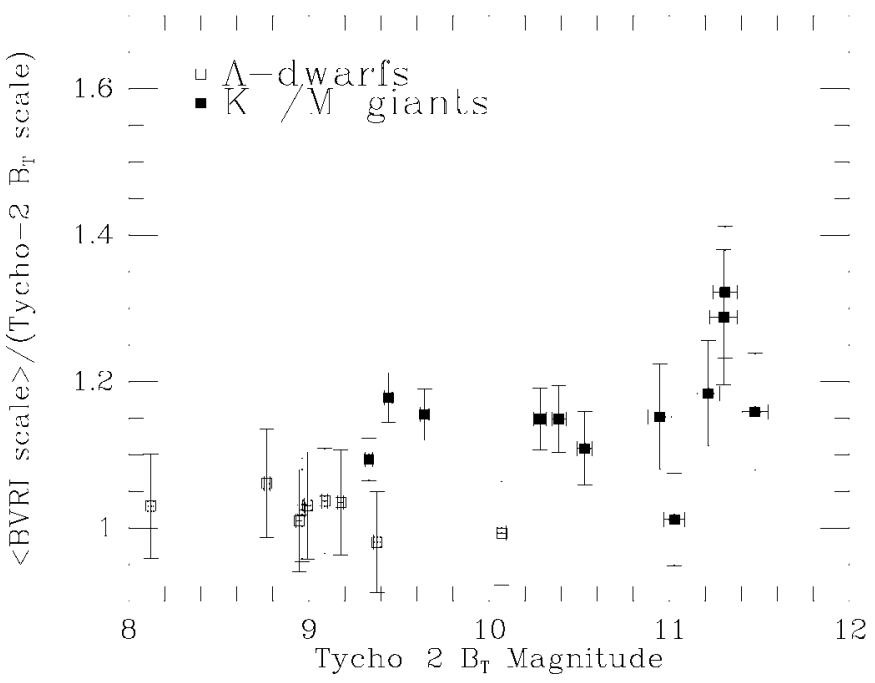

FIG. 12.-Ratio of ground-based to space-based optical photometric normalization factors for cool giants (filled squares) and A dwarfs (open squares) against Tycho-2's $B_{\mathrm{T}}$ magnitude.

$B_{\mathrm{T}}$ magnitudes between ratios of scale factors and $\mathrm{K}$ spectral class appears in Figure 14 along with the formal best-fit regression line. For Tycho-2, there is still a significant offset $(1.12 \pm 0.02)$, but the formal value for the slope is insignificantly different from unity $(0.01 \pm 0.01)$. Therefore, we consider it appropriate to rescale all $\mathrm{K}$ and $\mathrm{M}$ giant scale factors derived from Tycho- $2 B_{\mathrm{T}}$ data by a factor of $1.14 \pm 0.01$ (corresponding to the inverse-varianceweighted average of the $12 \mathrm{~K}$ giants' factors), thereby bringing them into alignment with $B V R I H_{p} V_{\mathrm{T}}$ normalization multipliers when we create absolute supertemplates. For those cool giants with Tycho-1 data but lacking Tycho-2 data (one star in our sample), we use the regression line in Figure 11 to correct $B_{\mathrm{T}}$ scale factors for Tycho-1 photometry of $\mathrm{K} / \mathrm{M}$ giants.

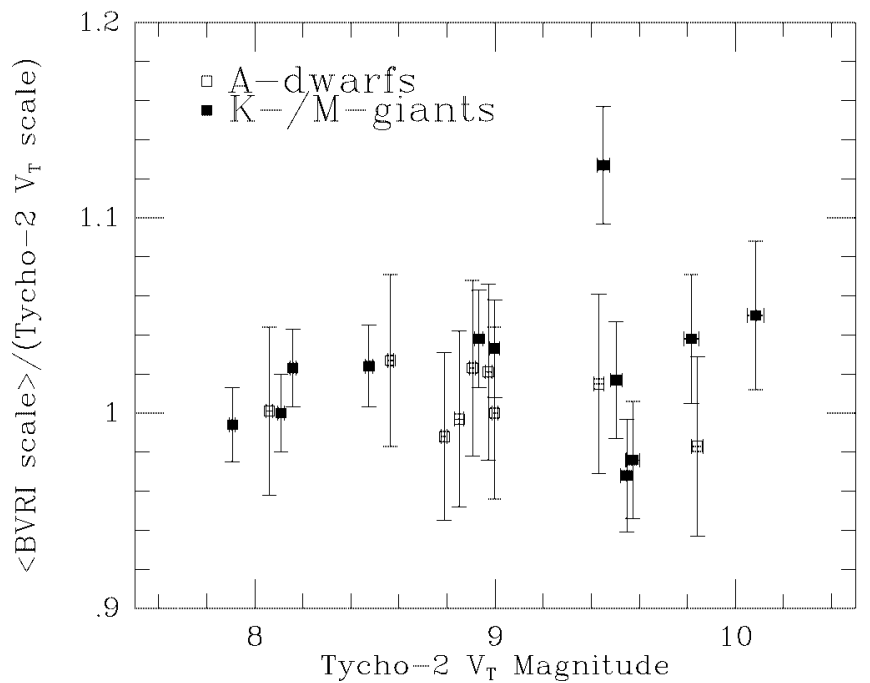

FIG. 13.-Same as Fig. 12, but for Tycho-2's $V_{\mathrm{T}}$ magnitude. The slope is statistically insignificant. 


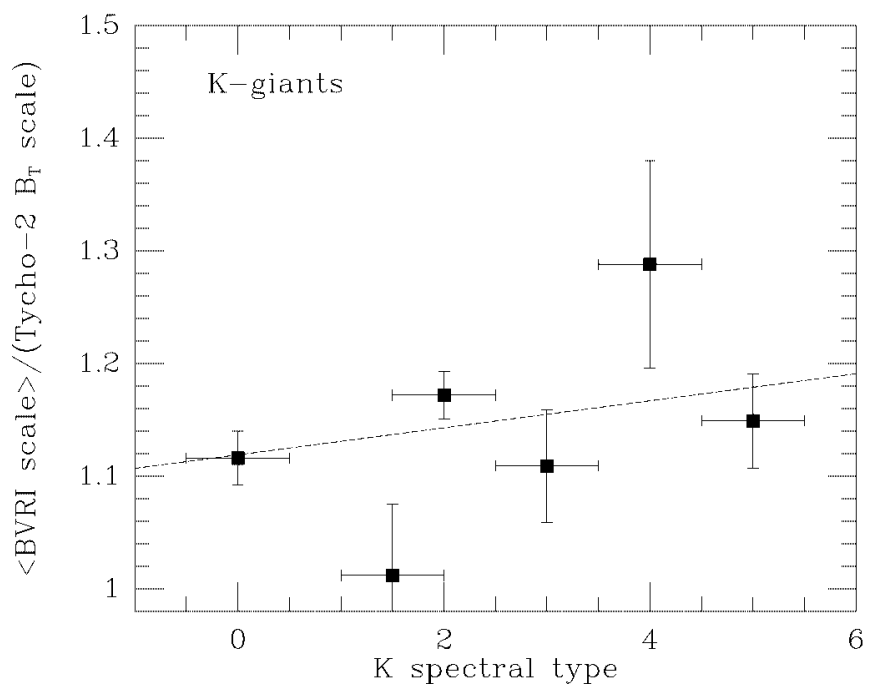

FIG. 14.- Ratio of ground-based to Tycho- $2 B_{\mathrm{T}}$ photometric normalization factors for $\mathrm{K}$ giants against $\mathrm{K}$ spectral class. The slope is statistically insignificant.

Advanced methods of data reduction, not available at the time Tycho-1 was processed, were applied to the Tycho-2 Catalogue (Høg et al. 2000); for example, all data series for a given star throughout the entire mission were co-added with appropriate weighting prior to deriving photometry from the accumulated signal. For low signal-to-noise signals in the Tycho star mappers, the gain in Tycho-2 was substantial, compared with Tycho-1. Known inherent biases in Tycho-1 are highlighted by Høg et al. (2000). Single transits of a star over the star mappers in which no obvious peak was identifiable were neglected in Tycho-1, leading to preferential selection of brighter transits of the same star $(\S 4.1$ of Høg et al. 2000). Second, in the presence of a changing background, the much longer series of sky samples used in Tycho-1 can lead to systematic offsets in the assessed background level ( $\$ 4.2$ of Høg et al. 2000). Both $V_{\mathrm{T}}$ and $H_{p}$ sample these cool stellar energy distributions well past the 4000 A break, where their spectra rise rapidly into the red, so there are no corresponding problems with cool stars in $V_{\mathrm{T}}$ or $H_{p}$.

We suggest that the phenomenon we see is due to the already known biases in Tycho-1 but, at least partly, to Malmquist bias, whereby the detection of faint red stars in $B_{\mathrm{T}}$ and, to a lesser extent, in $V_{\mathrm{T}}$ is enhanced by upward noise fluctuations, and this problem systematically worsens with later-type cool giants. Therefore, the $\mathrm{K} / \mathrm{M}$ giants appear brighter than reality, leading to smaller scale factors as stars become fainter and cooler. The improvements in the processing of Tycho-2 alleviate this problem and avoid the known problem of the "dramatic" "faint-end bias" intrinsic to Tycho-1 ( $\$ 7.1$ of Høg et al. 2000).
Bessell, M. S. 1979, PASP, 91, 589

1983, PASP, 95, 480

1990, PASP, 102, 118

2000, PASP, 112, 961

Bessell, M. S., Castelli, F., \& Plez, B. 1998, A\&A, 333, 231 (erratum 337, 321)

Cardelli, J. A., Clayton, G. C., \& Mathis, J. S. 1989, ApJ, 345, 245

Carter, B. S., \& Meadows, V. S. 1995, MNRAS, 276, 734

Cohen, M. 1993, AJ, 105, 1860

1998, AJ, 115, 2092

Cohen, M., Walker, R. G., Barlow, M. J., \& Deacon, J. R. 1992a, AJ, 104, 1650 (erratum 105, 2008 [1993])

Cohen, M., Walker, R. G., Carter, B., Hammersley, P., Kidger, M., \& Noguchi, K. 1999, AJ, 117, 1864 (Paper X)

Cohen, M., Walker, R. G., Jayaraman, S., Barker, E., \& Price, S. D. 2001, AJ, 121, 1180

Cohen, M., Walker, R. G., \& Witteborn, F. C. 1992b, AJ, 104, 2030 (erratum 105, 2008 [1993])

Cohen, M., Wheaton, W. A., \& Megeath, S. T. 2003, AJ, submitted

Cohen, M., Witteborn, F. C., Bregman, J. D., Wooden, D. H., Salama, A., \& Metcalfe, L. 1996a, AJ, 112, 241

Cohen, M., Witteborn, F. C., Carbon, D. F., Davies, J. K., Wooden, D. H., \& Bregman, J. D. 1996b, AJ, 112, 2274

Cohen, M., Witteborn, F. C., Walker, R. G., Bregman, J. D., \& Wooden, D. H. 1995 , AJ, 110, 275

Cousins, A. W. J. 1984, South African Astron. Obs. Circ., No. 8, 69

de Jager, C., \& Nieuwenhuijzen, H. 1987, A\&A, 177, 217

Drilling, J. S., \& Landolt, A. U. 1979, AJ, 84, 783

Egan, M. P., et al. 1999, The Midcourse Space Experiment Point Source Catalog Version 1.2 Explanatory Guide (Tech. Rep. AFRL-VS-TR1999-1522) (Hanscom AFB, MA: Air Force Res. Lab.)

ESA. 1997, The Hipparcos and Tycho Catalogues (SP-1200) (Noordwijk: ESA)

Fabricant, D., Cheimets, P., Caldwell, N., \& Geary, J. 1998, PASP, 110, 79

Fitzgerald, M. P. 1970, A\&A, 4, 234

Fitzpatrick, E. L. 1999, PASP, 111, 63

Fluks, M. A., Plez, B., Thé, P. S., de Winter, D., Westerlund, B. E., \& Steenman, H. C. 1994, A\&AS, 105, 311

\section{REFERENES}

Hauser, M. G., et al. 1998, ApJ, 508, 25

Hayes, D. S., \& Latham, D. W. 1975, ApJ, 197, 593

Høg, E., et al. 1997, A\&A, 323, L57

2000, A\&A, 357, 367

Houk, N., \& Swift, C. 1999, Michigan Catalogue of Two-dimensional Spectral Types for the HD Stars, Vol. 5 (Ann Arbor: Dept. Astron., Univ. Michigan)

Keenan, P. C., \& McNeil, R. C. 1989, ApJS, 71, 245

Kessler, M. F., et al. 1996, A\&A, 315, L27

Kurucz, R. L. 1993a, CD-ROM 13, ATLAS9 Stellar Atmosphere Programs and $2 \mathrm{~km} / \mathrm{s}$ Grid (Cambridge: Smithsonian Astrophys. Obs.) . 1993b, CD-ROM 18, SYNTHE Spectrum Synthesis Programs and

Line Data (Cambridge: Smithsonian Astrophys. Obs.)

Landolt, A. U. 1973, AJ, 78, 959 1983, AJ, 88, 439 1992, AJ, 104, 340

Menzies, J. W. 1989, MNRAS, 237, 21P

Menzies, J. W., Marang, F., Laing, J. D., Coulson, I. M., \& Engelbrecht, C. A. 1991, MNRAS, 248, 642

Mill, J. D., et al. 1994, J. Spacecr. Rockets, 31, 900

Murakami, H., et al. 1996, PASJ, 48, L41

O’Donnell, J. E. 1994, ApJ, 422, 158

Oke, J. B., \& Gunn, J. E. 1983, ApJ, 266, 713

Perry, C. L., \& Johnston, L. 1982, ApJS, 50, 451

Perry, C. L., Johnston, L., \& Crawford, D. L. 1982, AJ, 87, 1751

Pickles, A. J. 1998, PASP, 110, 863

Price, S. D., Egan, M. P., Carey, S. J., Mizuno, D. R., \& Kuchar, T. A. 2001, AJ, 121, 2819

Rieke, G. H., \& Lebofsky, M. J. 1985, ApJ, 288, 618

Straižys, V. 1992, Multicolor Stellar Photometry (Tucson: Pachart)

van Leeuwen, F. 1997, Space Sci. Rev., 81, 201

Walker, R. G., \& Cohen, M. 2002, Walker-Cohen Atlas of Calibrated Spectra: Explanatory Supplement to Release 4.0 (contract. rep. to Air Force Res. Lab., contract F19628-98-C-0047)

Witteborn, F. C., Cohen, M., Bregman, J. D., Heere, K. R., Greene, T. P., \& Wooden, D. H. 1995, in ASP Conf. Ser. 73, Airborne Astronomy Symposium on the Galactic Ecosystem, ed. M. R. Haas, J. A. Davidson, \& E. F. Erickson (San Francisco: ASP), 573 